\title{
Phytoplankton of the Morava and Dyje Rivers in spring and summer 2006
}

\author{
Petr HAŠLER ${ }^{1}$, František HINDÁK ${ }^{2} \&$ Alica HINDÁKOVÁ ${ }^{2}$ \\ ${ }^{1}$ Department of Botany, Faculty of Sciences, Palacký University, Šlechitelu 11, CZ-78371 Olomouc-Holice, \\ Czech Republic; e-mail:petr.hasler@upol.cz. \\ ${ }^{2}$ Institute of Botany, Slovak Academy of Sciences, Dúbravská cesta 14, SK-84523 Bratislava, Slovakia; e-mail: \\ frantisek.hindak@savba.sk, alica.hindakova@savba.sk
}

\begin{abstract}
Species composition and abundance of the phytoplankton of the Morava and Dyje Rivers are evaluated. Samples were collected at Olomouc, Lanžhot, Devín (the Morava River) and at Pohansko (the Dyje River) in May and August 2006. Both concentrated samples using plankton net and free water were taken at each sampling point. Environmental variables such as $\mathrm{pH}$, temperature, conductivity and dissolved oxygen were also measured. Altogether 542 species and infraspecific taxa of cyanobacteria and algae were identified. Collected data of diversity and abundance were statistically analyzed using NCSS software and Canoco for Windows. Significant differences between sampling sites and sampling time were observed. A complete list of species found at investigated sites is supplied.
\end{abstract}

Key words: Morava and Dyje Rivers, potamoplankton, biodiversity, abundance, Czech Republic, Slovak Republic

\section{Introduction}

The Morava River belongs to the most important rivers together with the Labe and Vltava in the Czech Republic and is the first major left - side tributary of the Danube River in Slovakia near the point of its entry into Pannonian Lowland. It is a typical regulated lowland river draining about $24,000 \mathrm{~km}^{2}$ of the Moravian Region of the Czech Republic and parts of Western Slovakia and the NE part of Lower Austria. The river can be divided into three major parts. The border between upper and middle stretch forms left side tributary of the Bečva River with river basin of about $1,627 \mathrm{~km}^{2}$, which is one of the main rivers flowing through the NE Moravia. The bioseston in the upper stretch mainly consists of drifting benthic diatoms and only low proportion of true planktonic species. Over the past century, the level of pollution has been changed as a result of rapid human development, including paper mill effluents and agricultural activities. The Dyje River forms the border between the lowermost and middle stretch. Important reservoires such as Nové Mlýny, Znojmo and Vranov have dammed the river. In the middle stretch with numerous left - and right - side tributaries as well as meanders along its whole length planktonic diatoms, green algae and flagellates prevail over tychoplankton. Generally, dam reservoirs and their impoundments as well as river bed regulations have led to changes in the species composition of potamoplankton (cf. MARVAN \& HETEŠA 2000, MARVAN et al. 2004, HINDÁK et al. 2006).

Previous investigations in the lowermost stretch of the Morava River showed an extremely broad spectrum of algae from different taxonomic groups including 206 genera with 692 species and infraspecific taxa (HINDÁK 1977 1990, HINDÁK et al 2006, HINDÁK \& HINDÁKOVÁ 1997, 1998, 2004, HINDÁKOVÁ 1994, HINDÁK \& MAKOVINSKÁ 1996). Several new taxa from the Morava and Danube Rivers were described, e.g. cyanophytes Cyanocatena planctonica Hindák 1975, C. verrucosa HINDÁK 2002, Radiocystis aphanothecoidea HINDÁK 1996, Cyanogranis basifixa HINDÁK 1982, C. libera HINDÁK 2002, Romeria simplex (HINDÁK 1975) HINDÁK 1988, as well as chlorophytes, e.g. Coenochloris astroidea HINDÁK 1988, Neocystis diplococca (HINDÁK 1978) HINDÁK 1988, Neodesmus danubialis HINDÁK 1976, Raphidocelis sigmoidea HINDÁK 1977 and Marvania geminata HINDÁK 1976 (see also MARVAN et al. 2004). The upper Morava River was sampled occasionally and collected data did not allow a full comparison with the lower stretch possible (HETEŠA \& MARVAN 1984, 1987, 1997, MARVAN et al. 2004). Last 
investigations on diversity and abundance of phytoplankton in the Morava and Dyje Rivers were carried out in November 2005 by HINDÁK et al. (2006). It was found the significant differences among sampling sites located in the upper, middle and lowermost stretches.

\section{Methods and localities}

The phytoplankton samples of the Morava River were collected in May 24 and August 16, 2006, similarly as from the Dyje River (Fig. 1) using plankton net (mesh size $10 \mu \mathrm{m}$ ). Three sampling points were choosen at the Morava River as follows: Olomouc $\left(232^{\text {nd }}\right.$ river $\mathrm{km}$, average discharge $\left.27.1 \mathrm{~m}^{3} \cdot \mathrm{s}^{-1}\right)$, Lanžhot $\left(78^{\text {th }}\right.$ river $\mathrm{km}$, average discharge $54 \mathrm{~m}^{3} . \mathrm{s}^{-1}$ ), Devín (average discharge $120 \mathrm{~m}^{3} \cdot \mathrm{s}^{-1}$, near the confluence with the Danube River) and one at the Dyje River: Pohansko (the lowermost river stretch near the confluence with the Morava River, average discharge $\left.41.7 \mathrm{~m}^{3} \cdot \mathrm{s}^{-1}\right)$. Samples for the quantitative analysis were immediately preserved by formaldehyde (preserved sample $\sim 2 \%$ solution). Environmental variables such as $\mathrm{pH}$, conductivity, temperature and dissolved oxygen were measured in situ using instruments by WTW (Tab. 1). All live samples were analysed no later than $24 \mathrm{~h}$ after sampling by light microscopes Leitz Diaplan and Zeiss Axio Imager equiped with HRc camera. Planktonic microorganisms were identified according to monographs of HINDÁK (1977, 1980, 1984, 1988, 1990, 1996, 2001), HUBER-PESTALOZZI (1955), KOMÁREK \& ANAGNOSTIDIS (1998, 2005), KRAMMER \& LANGE-BERTALOT (1986, 1988, 1991a, b) and WOŁOWSKI \& HINDÁK (2005). Quantitative plankton analyses are based on counts of organisms in a Bürker chamber after samples concentration by ultrafiltration (pores size $1.2 \mu \mathrm{m}$ ). At least 400 organisms were canonical correspondence analysis (CCA, ŠMILAUER \& TER BRAAK 1998). We used biplot scaling ( $\left.\mathrm{L}^{\wedge} \mathrm{a}\right)$ focused on inter-species distances. Species data were $\log$ transformed $\left[\mathrm{Y}^{\prime}=\log \left(\mathrm{A}^{*} \mathrm{Y}+\mathrm{B}, \mathrm{A}=1, \mathrm{~B}=1\right)\right]$ with downweighting of rare species. Monte-Carlo permutation test with significance of first ordination axis under reduced model (499 permutations) was performed. Both automatic and manual selection with unrestricted permutations were used to test marginal and conditional effects of measured environmental variables. counted in all samples. Dissimilarity among sampling sites with respect to abundance of counted algal groups was evaluated with hierarchical clustering analysis (Wards' method, NCSS software). Influence of environmental variables such as temperature, $\mathrm{pH}$ and conductivity on species data was evaluated with



Fig. 1. Draining area of the Morava River and sampling sites (dark circles).

\section{Results}

\section{Phytoplankton abundance}

The highest abundance of phytoplankton was counted at Devín $\left(124,627\right.$ cells.ml $\left.^{-1}\right)$ in spring, while the lowest one was found at Olomouc also in spring $\left(6,414\right.$ cells.ml $\left.^{-1}\right)$ (Tab. 2). The most abundant groups were found cyanobacteria, diatoms and green coccal algae in all samples forming the majority of biomass, but in different ratios (spring: $26: 41: 22 \%$, summer $34: 37$ : $17 \%$, average ratios from all investigated localities). Diatoms dominated in all investigated samples, with the highest portion in spring at Devín (Fig. 3). In comparison with spring, the lower abundance of all phytoplankton was found in summer at Devín as a consequence of a flood in the beginning of August when diatoms and cyanobacteria formed an important portion of phytoplankton. Coccal green algae represented approximately $20 \%$ of phytoplankton with maximum in spring (Fig. 3). The other counted groups of algae such as Cryptophyceae, Chrysophyceae, green flagellates, Ulotrichales, Conjugatophyceae and Euglenophyceae were usually found on low level. Results of hierarchical clustering analysis (Ward's method) based on 
abundances of counted groups of cyanobacteria and algae show differences among investigated sites (Figs 2A, B). Both spring and summer samples could be divided into two major groups containing the Morava River at Olomouc and the Dyje River with lower abundance, and the Morava River at Lanžhot and Devín with higher abundance of phytoplankton.
Table 1. Measured environmental variables: Tem - Temperature ${ }^{\circ} \mathrm{C}, \mathrm{pH}$, Con - Conductivity $/ \mu \mathrm{S} . \mathrm{cm}^{-1}$, OxA-Oxygen-abs $/ \mathrm{mg} . \mathrm{l}^{-1}$, OxR - Oxygen-rel 1\%, OL - Morava at Olomouc, LA - Morava at Lanžhot, DE - Morava at Devín, DY - Dyje at Pohansko.

\begin{tabular}{lcccccccc}
\hline & \multicolumn{4}{c}{ May } & \multicolumn{4}{c}{ August } \\
& OL & LA & DE & DY & OL & LA & DE & DY \\
\hline Tem & 7.5 & 8.9 & 8.9 & 9.4 & 14.4 & 17.2 & 16.5 & 17.3 \\
$\mathbf{p H}$ & 7.65 & 8.07 & 7.67 & 8.27 & 7.39 & 8.19 & 8.05 & 8.96 \\
$\mathbf{C o n}$ & 334 & 668 & 651 & 581 & 298 & 523 & 594 & 504 \\
$\mathbf{O x A}$ & 8.5 & 9.2 & - & 8.4 & 8.8 & 8.4 & 8.6 & 8.6 \\
$\mathbf{O x R}$ & 100 & 106 & - & 93 & 98 & 100 & 102 & 102 \\
$\mathbf{N O}_{3}{ }^{-}$ & - & - & - & - & 9.9 & 9.9 & 9.8 & 11.8 \\
$\mathbf{N H}_{4}{ }^{+}$ & - & - & - & - & 0.07 & 0.03 & 0.03 & 0.18 \\
$\mathbf{P O}_{4}{ }^{3-}$ & - & - & - & - & 0.23 & 0.16 & 0.35 & 0.52 \\
\hline
\end{tabular}

Table 2. Phytoplankton abundance in counted major groups $\left(\right.$ cells.ml ${ }^{-1}$ )

\begin{tabular}{|c|c|c|c|c|c|}
\hline Groups & $\begin{array}{l}\text { Locality/ } \\
\text { Month }\end{array}$ & OL & LA & DE & DY \\
\hline \multirow[t]{2}{*}{ Cyanobacteria } & May & 1463 & 6343 & 34632 & 3891 \\
\hline & August & 12549 & 16764 & 16020 & 14680 \\
\hline \multirow[t]{2}{*}{ Cryptophyceae } & May & 160 & 426 & 452 & 213 \\
\hline & August & 264 & 534 & 445 & 356 \\
\hline \multirow[t]{2}{*}{ Chrysophyceae } & May & 186 & 1812 & 8214 & 0 \\
\hline & August & 712 & 1157 & 267 & 267 \\
\hline \multirow[t]{2}{*}{ Coscinodiscales } & May & 1170 & 13165 & 36408 & 5064 \\
\hline & August & 5340 & 25543 & 15664 & 8360 \\
\hline \multirow[t]{2}{*}{ Naviculales } & May & 479 & 2237 & 13776 & 906 \\
\hline & August & 3916 & 2403 & 2403 & 2072 \\
\hline \multirow[t]{2}{*}{ Volvocales } & May & 426 & 924 & 1554 & 373 \\
\hline & August & 665 & 712 & 1335 & 798 \\
\hline \multirow[t]{2}{*}{ Chlorococcales } & May & 1702 & 6129 & 27750 & 3944 \\
\hline & August & 5054 & 8366 & 11392 & 5607 \\
\hline \multirow[t]{2}{*}{ Ulotrichales } & May & 267 & 380 & 313 & 88 \\
\hline & August & 534 & 534 & 614 & 176 \\
\hline \multirow[t]{2}{*}{ Conjugatophyceae } & May & 534 & 801 & 640 & 580 \\
\hline & August & 1068 & 1602 & 1273 & 1149 \\
\hline \multirow[t]{2}{*}{ Euglenophyceae } & May & 27 & 160 & 888 & 320 \\
\hline & August & 801 & 2670 & 1602 & 931 \\
\hline \multirow[t]{2}{*}{$\Sigma$} & May & 6414 & 32377 & 124627 & 15379 \\
\hline & August & 30903 & 60285 & 51015 & 34396 \\
\hline
\end{tabular}
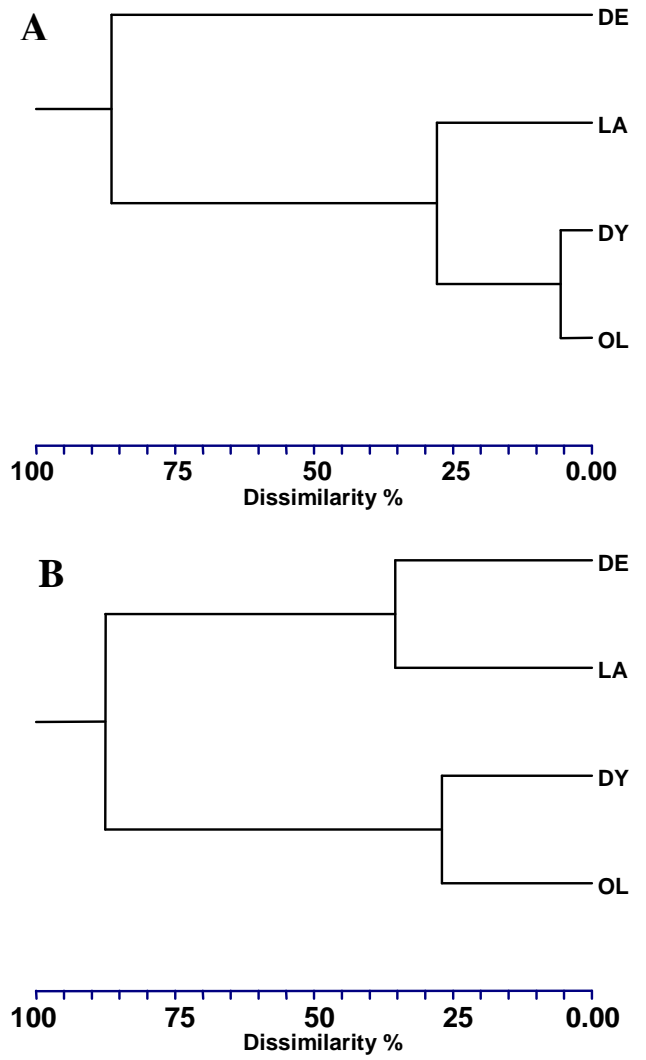

Fig. 2. Dendrograms of hierarchical clustering (Ward's method) based on abundances of counted groups of cyanobacteria and algae: A - May, B August. 


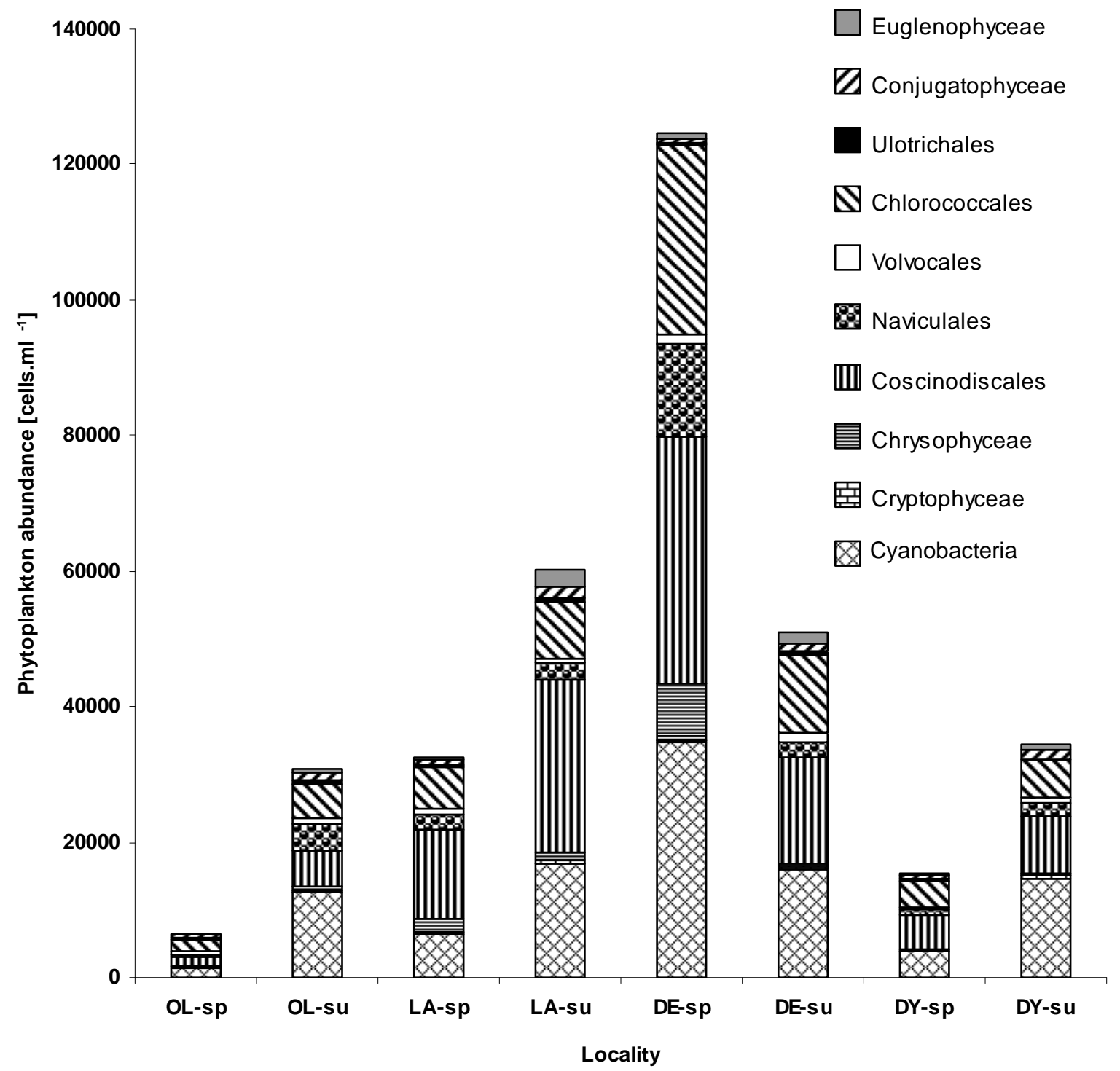

Fig. 3. Abundance of phytoplankton: OL - Morava River at Olomouc, LA - Morava river at Lanžhot, DE - Morava river at Devín, DY - Dyje River at Pohansko; suffix: sp-sampling in spring (May), su-sampling in summer (August).

\section{Phytoplankton species composition}

Altogether 542 taxa of cyanobacteria and algae were recorded at the investigated sites during our study (Tab. 3). The highest species richness were found in cyanobacteria with 53 taxa, in diatoms with 189 taxa and in green coccal algae with total number of 109 taxa (Tab. 5). The lowest richness was found in the Dyje River. The Morava River at Olomouc can be characterised by dominance of pennate diatoms in contrast to lower stretch (Lanžhot and Devín) dominated by green algae.

The canonical correspondence analysis (CCA) was performed to test influence of measured environmental variables on species composition of cyanobacteria and algae (Figs 4, 5). The Morava River at Olomouc was significantly different from other localities, they are influenced by Nové Mlýny Reservoir $(\mathrm{F}=1.316, \mathrm{P}=0.032$, Monte-Carlo permutation test for the first axis, reduced model, 499 permutations). Conductivity was found as an important factor influencing phytoplankton composition among sampling sites especially in spring $(\mathrm{F}=1.78, \quad \mathrm{P}=0.008)$, while summer phytoplankton was more influenced by gradient of temperature. Significance of measured $\mathrm{pH}$ was very low with respect to small differences among sampling sites (Tab. 4). Marginal effects summarize variance of individual environmental variables explaining singly (lambda-1). Conditional effects explain environmental variables in the model together (lambda-A). Using CCA four groups of cyanobacteria and algae can be recognized: 
Group I: Species occurring at Olomouc more frequently than in the lower Morava stretch As it follows from statistical reports (Fig. 5), the phytoplankton consist of pennate diatoms, several green flagellates, euglenophytes and coccal chlorophytes. Pennate diatoms are predominantly formed by benthic species (Fig. $5)$.

\section{Group II: Species commonly occurring at Lanžhot and Devín in spring}

Phytoplankton in the Morava River both at Lanžhot and Devín was rich in cyanobacteria, centric diatoms, coccal chlorophytes and particularly flagellates (Fig. 5).

\section{Group III: Species commonly occurring at Lanžhot and Devín in summer}

The highest richness at Lanžhot and Devín was found in the summer period. Together with the most developed coccal green algae also occurred cyanobacteria, pennate diatoms, euglenophytes and tetrasporalean algae.

\section{Group IV: Group of commonly occurring species}

The majority of species found in the Morava and Dyje Rivers is commonly occurring cyanophytes and algae in this region. There is no doubt that species of this group influenced total diversity of the Morava and Dyje Rivers (Fig. 5).

Table 3. List of species recorded in the Morava River at Olomouc (OL), Lanžhot (LA), Devín (DE) and in the Dyje River (DY), a -sampling May, b-sampling August. Taxa marked with asterisk (*) are new records for the Morava River.

\begin{tabular}{|c|c|c|c|c|}
\hline & $\mathbf{O L}$ & $\mathbf{L A}$ & $\mathbf{D E}$ & DY \\
\hline \multicolumn{5}{|l|}{ CYANOPHYCEAE } \\
\hline \multicolumn{5}{|l|}{ Chroococcales } \\
\hline Aphanocapsa incerta (LEMMERM.) CRONBERG et KOMÁREK & $\mathrm{ab}$ & $\mathrm{ab}$ & $\mathrm{ab}$ & \\
\hline Aphanocapsa grevillei (BERKELEY) RABENH. & & & $\mathrm{b}$ & $\mathrm{b}$ \\
\hline Aphanocapsa holsatica (LEMMERM.) CRONBERG et KOMÁREK & $\mathrm{b}$ & & $\mathrm{b}$ & \\
\hline Aphanocapsa minima Migula & & & $\mathrm{b}$ & \\
\hline Aphanocapsa parasitica (KÜTZ.) KOMÁREK et ANAGN. & & a & & \\
\hline Aphanothece bachmanii KOMÁRK.-LEGN. et CRONBERG & & & $\mathrm{b}$ & \\
\hline Aphanothece clathrata W. et G.S. WEST & & $\mathrm{ab}$ & & $\mathrm{ab}$ \\
\hline Aphanothece floccosa (ZALESSKY) CRONBERG et KOMÁREK & & & $a b$ & \\
\hline Chroococcus limneticus LEMMERM. & $a b$ & $\mathrm{ab}$ & $a b$ & $\mathrm{ab}$ \\
\hline Coelomoron pusillum (VAN GOOR) KOMÁREK & $a b$ & & $a b$ & $a b$ \\
\hline Cyanocatena planctonica HINDÁK & & & $a b$ & \\
\hline *Cyanocatenula calyptrata JoOsTEN & & & $a b$ & \\
\hline Cyanogranis basifixa HINDÁK & $\mathrm{b}$ & $\mathrm{ab}$ & $a b$ & \\
\hline Cyanogranis ferruginea (WAWRIK) HINDÁK & $a b$ & $\mathrm{ab}$ & $a b$ & $\mathrm{ab}$ \\
\hline Cyanogranis libera HINDÁK & & $\mathrm{ab}$ & $a b$ & $\mathrm{ab}$ \\
\hline Merismopedia ferrophila HINDÁK & $\mathrm{b}$ & & & \\
\hline Merismopedia glauca (EHRENB.) KüTZ. & & & $\mathrm{ab}$ & \\
\hline Merismopedia tenuissima LEMMERM. & $a b$ & $\mathrm{ab}$ & & \\
\hline Merismopedia warmingiana LAGERH. & & $\mathrm{ab}$ & $a b$ & $a b$ \\
\hline Microcystis aeruginosa (KüTZ.) KüTZ. & $a b$ & $\mathrm{ab}$ & $a b$ & $a b$ \\
\hline Microcystis flos-aquae (WITTR.) KIRCHN. & & $\mathrm{ab}$ & $a b$ & \\
\hline Microcystis ichhtyoblabe KüTZ. & $a b$ & $\mathrm{ab}$ & $a b$ & $a b$ \\
\hline Microcystis novacekii (KOMÁREK) COMPÈRE & & $\mathrm{ab}$ & $a b$ & $?$ \\
\hline Microcystis viridis (A.BR.) LEMMERM. & $\mathrm{b}$ & $\mathrm{b}$ & & $\mathrm{b}$ \\
\hline Microcystis wesenbergii (KOMÁREK) KOMÁREK & $a b$ & $a b$ & $a b$ & $a b$ \\
\hline Pannus spumosus B. HICKEL & $a b$ & $\mathrm{ab}$ & $a b$ & $\mathrm{ab}$ \\
\hline Romeria okensis (MEYER) HINDÁK & $\mathrm{b}$ & $\mathrm{b}$ & $\mathrm{b}$ & \\
\hline Snowella atomus KOMÁREK et HINDÁK & $\mathrm{b}$ & & & \\
\hline
\end{tabular}




\begin{tabular}{|c|c|c|c|c|}
\hline Cont. Table 3 & $\mathbf{O L}$ & $\overline{\mathbf{L A}}$ & $\overline{\mathbf{D E}}$ & $\overline{D Y}$ \\
\hline Snowella litoralis (HÄYRÉN) KOMÁREK et HINDÁK & $\mathrm{ab}$ & $\mathrm{ab}$ & $\mathrm{ab}$ & $\mathrm{ab}$ \\
\hline Woronichinia naegeliana (UNGER) ELENKIN & $\mathrm{ab}$ & $a b$ & $a b$ & $a b$ \\
\hline \multicolumn{5}{|l|}{ Oscillatoriales } \\
\hline Geitlerinema sp. & $\mathrm{ab}$ & $\mathrm{ab}$ & $a b$ & $a b$ \\
\hline Limnothrix redekei (GOOR) MEFFERT & $\mathrm{ab}$ & $\mathrm{ab}$ & $a b$ & \\
\hline Oscillatoria janus SKUјА & $\mathrm{b}$ & $\mathrm{ab}$ & $a b$ & \\
\hline Oscillatoria limosa AGARDH ex GOMONT & $\mathrm{ab}$ & & & $a b$ \\
\hline Oscillatoria tenuis C. AGARDH ex GOMONT & $\mathrm{ab}$ & & & \\
\hline $\begin{array}{l}\text { Planktolyngbya limnetica (LEMMERM.) KOMÁRK.-LEGN. et } \\
\text { CRONBERG }\end{array}$ & $\mathrm{ab}$ & $\mathrm{ab}$ & $a b$ & \\
\hline Planktothrix agardhii (GOMONT) ANAGN. et KOMÁREK & $\mathrm{ab}$ & $\mathrm{ab}$ & $\mathrm{ab}$ & $\mathrm{ab}$ \\
\hline Planktothrix cryptovaginata (ŠKORBATOV) ANAGN. et KOMÁREK & & & & $\mathrm{b}$ \\
\hline Planktothrix isothrix (SKUJA) KOMÁREK et KOMÁRKOVÁ & & $\mathrm{b}$ & & $\mathrm{b}$ \\
\hline Pseudanabaena catenata LAUTERBORN & $\mathrm{ab}$ & $\mathrm{ab}$ & $\mathrm{ab}$ & \\
\hline Pseudanabaena limnetica (LEMMERM.) KOMÁREK & $\mathrm{ab}$ & $\mathrm{ab}$ & $\mathrm{ab}$ & \\
\hline Pseudanabaena mucicola (NAUMANN et Hub.-PEST.) Bourr. & $\mathrm{ab}$ & $\mathrm{ab}$ & $\mathrm{ab}$ & $\mathrm{ab}$ \\
\hline \multicolumn{5}{|l|}{ Nostocales } \\
\hline Anabaena circinalis RABENH. ex BORNET et FLAHAUT & & $\mathrm{a}$ & a & \\
\hline Anabaena crassa (LEMMERM.) KOM.-LEGN. et CRONBERG & & $\mathrm{ab}$ & $a b$ & \\
\hline Anabaena compacta (NYGAARD) HICKEL & & $\mathrm{ab}$ & $a b$ & $\mathrm{ab}$ \\
\hline Anabaena flos-aquae (LyngB.) BrÉB. ex BORNET et FlAHAUlt & $\mathrm{ab}$ & $\mathrm{ab}$ & $\mathrm{ab}$ & $\mathrm{ab}$ \\
\hline Anabaena perturbata HILL & & $\mathrm{b}$ & & \\
\hline Anabaena planctonica BRUNNTH. & $\mathrm{ab}$ & $\mathrm{ab}$ & $\mathrm{ab}$ & \\
\hline Anabaenopsis elenkinii BRUNNTH. & $\mathrm{ab}$ & $\mathrm{ab}$ & $\mathrm{ab}$ & \\
\hline Aphanizomenon gracile (LEMMERM.) LEMMERM. & & & $a b$ & $\mathrm{ab}$ \\
\hline Aphanizomenon flos-aquae (L.) RALFS ex BORNET et FLAHAUT & $\mathrm{b}$ & & $\mathrm{b}$ & $\mathrm{b}$ \\
\hline Aphanizomenon issachenkoi (UsSACZEW) PROSHK.-LAVR. & $\mathrm{b}$ & $\mathrm{b}$ & $\mathrm{b}$ & $\mathrm{b}$ \\
\hline Raphidiopsis mediterranea SKUJA & & $\mathrm{ab}$ & $\mathrm{ab}$ & \\
\hline \multicolumn{5}{|l|}{ RHODOPHYCEAE } \\
\hline Chantransia sp. & & $\mathrm{b}$ & & \\
\hline \multicolumn{5}{|l|}{ CHRYSOPHYCEAE } \\
\hline Chrysococcus diaphanus SKUJA & & & $\mathrm{ab}$ & \\
\hline Chrysococcus rufescens G. A. KLEBS & $\mathrm{ab}$ & & $\mathrm{ab}$ & $\mathrm{ab}$ \\
\hline Dinobryon divergens О. Е. ІмноF & & $\mathrm{ab}$ & $\mathrm{ab}$ & \\
\hline Hymenomonas roseola $\mathrm{F}$. STEIN & & & $\mathrm{ab}$ & $\mathrm{ab}$ \\
\hline Mallomonas robusta MATVIENKO & & & a & \\
\hline Mallomonas sp. & $\mathrm{ab}$ & $\mathrm{ab}$ & $\mathrm{ab}$ & $\mathrm{ab}$ \\
\hline Ochrobium tectum PERFILIEV. & & & $\mathrm{b}$ & \\
\hline Pseudokephyrion entzii W. CONRAD & & & $\mathrm{ab}$ & \\
\hline Synura sp. & $\mathrm{ab}$ & $\mathrm{ab}$ & $\mathrm{ab}$ & \\
\hline \multicolumn{5}{|l|}{ BACILLARIOPHYCEAE } \\
\hline \multicolumn{5}{|l|}{ Coscinodiscales } \\
\hline Acanthoceras zachariasii (BRUN) SIMONSEN & $\mathrm{ab}$ & $\mathrm{ab}$ & $a b$ & $\mathrm{ab}$ \\
\hline Actinocyclus normanii (W. GREG. ex GREV.) HUST. & & & & $\mathrm{ab}$ \\
\hline Aulacoseira ambigua (GRUNOw) SIMONSEN var. ambigua & $\mathrm{ab}$ & $\mathrm{ab}$ & $\mathrm{ab}$ & $\mathrm{ab}$ \\
\hline Aulacoseira ambigua var. curvata (GRUNOW) SIMONSEN & $\mathrm{ab}$ & $?$ & $?$ & $?$ \\
\hline Aulacoseira granulata var. curvata GRUNOW & $\mathrm{ab}$ & $\mathrm{ab}$ & $\mathrm{ab}$ & $\mathrm{ab}$ \\
\hline Aulacoseira granulata (EHRENB.) SIMONSEN var. granulata & $\mathrm{ab}$ & $\mathrm{ab}$ & $\mathrm{ab}$ & $\mathrm{ab}$ \\
\hline Aulacoseira muzzanensis (F. MEISTER) KRAMMER & $\mathrm{ab}$ & $\mathrm{ab}$ & $\mathrm{ab}$ & $\mathrm{ab}$ \\
\hline Aulacoseira subarctica (O. MÜLL.) E. Y. HAW. & $\mathrm{ab}$ & $\mathrm{ab}$ & $\mathrm{ab}$ & $\mathrm{ab}$ \\
\hline
\end{tabular}




\begin{tabular}{|c|c|c|c|c|}
\hline Cont. Table 3 & $\overline{\mathbf{O L}}$ & $\overline{\mathbf{L A}}$ & $\overline{D E}$ & $\overline{D Y}$ \\
\hline Cyclotella atomus HuST. & $\mathrm{ab}$ & $\mathrm{ab}$ & $\mathrm{ab}$ & $\mathrm{ab}$ \\
\hline Cyclotella meneghiniana KüTZ. & $a b$ & $\mathrm{ab}$ & $a b$ & $a b$ \\
\hline Cyclotella distinguenda HusT. & $a b$ & & & \\
\hline Cyclotella ocellata PANT. & $a b$ & $a b$ & $a b$ & $a b$ \\
\hline Cyclotella pseudocomensis SCHEFFLER & & & $\mathrm{ab}$ & \\
\hline Cyclotella pseudostelligera Hust. & $a b$ & & & \\
\hline Cyclotella quadrijuncta (SCHRÖT.) KEISSL. & $a b$ & & & \\
\hline Cyclotella stelligera CLEVE et GRUNOW & $a b$ & & $\mathrm{ab}$ & \\
\hline Cyclotella stelligeroides HusT. & $\mathrm{b}$ & $\mathrm{b}$ & $\mathrm{b}$ & $\mathrm{b}$ \\
\hline Cyclotella woltereckii Hust. & $a b$ & & $\mathrm{ab}$ & \\
\hline Cyclotella sp. & $\mathrm{a}$ & & & \\
\hline Cyclostephanos delicatus (GENKAL) CASPER et SCHEFFLER & $\mathrm{ab}$ & $a b$ & $\mathrm{ab}$ & $a b$ \\
\hline Cyclostephanos dubius (FRICKE) ROUND & $\mathrm{ab}$ & $\mathrm{ab}$ & $\mathrm{ab}$ & $a b$ \\
\hline $\begin{array}{l}\text { Cyclostephanos invisitatus (M. H. HoHN et Hellerman) E. C. } \\
\text { THER., STOERMER et HÅK. }\end{array}$ & $\mathrm{ab}$ & $\mathrm{ab}$ & $\mathrm{ab}$ & \\
\hline Melosira varians $\mathrm{C}$. AGARDH & $a b$ & $\mathrm{ab}$ & $a b$ & $a b$ \\
\hline Skeletonema potamos (C. I. WEBER) HASLE & $\mathrm{ab}$ & $a b$ & $\mathrm{ab}$ & $a b$ \\
\hline Stephanodiscus alpinus HusT. & & & $\mathrm{b}$ & \\
\hline Stephanodiscus binderanus (KÜTZ.) WILLI KRIEG. & $a b$ & $a b$ & $\mathrm{ab}$ & $a b$ \\
\hline Stephanodiscus hantzschii GRUNOw f. hantzschii & $a b$ & & $\mathrm{ab}$ & $a b$ \\
\hline Stephanodiscus hantzschii f. tenuis (HusT.) НА̊K. & $a b$ & $a b$ & $\mathrm{ab}$ & $a b$ \\
\hline Stephanodiscus sp. & $a b$ & $a b$ & $\mathrm{ab}$ & \\
\hline Thalassiosira duostra PIENAAR et PIETERSE & $a b$ & $\mathrm{ab}$ & $\mathrm{ab}$ & $a b$ \\
\hline Thalassiosira pseudonana HASLE et HEIMDAL & $\mathrm{ab}$ & $a b$ & $\mathrm{ab}$ & \\
\hline Thalassiosira weissflogii (GRUNOw) G. A. FRYXELL et HASLE & & & $\mathrm{ab}$ & \\
\hline \multicolumn{5}{|l|}{ Naviculales } \\
\hline Achnanthes exigua GRUNOw & & & & $a b$ \\
\hline Achnanthes hungarica (GRUNOW) GRUNOW & $\mathrm{ab}$ & & $\mathrm{ab}$ & $a b$ \\
\hline Achnanthes cf. laevis OESTRUP & $a b$ & & & \\
\hline Achnanthes lanceolata (BRÉB. ex KÜTZ.) GRUNOW var. lanceolata & $\mathrm{ab}$ & $\mathrm{ab}$ & $\mathrm{ab}$ & $a b$ \\
\hline Achnanthes lanceolata var. rostrata (OESTRUP) HUST. & $\mathrm{ab}$ & & $\mathrm{ab}$ & \\
\hline Achnanthes minutissima KüTZ. & $a b$ & $\mathrm{ab}$ & $\mathrm{ab}$ & \\
\hline Achnanthes ploenensis HUST. & & & $\mathrm{ab}$ & $a b$ \\
\hline Achnanthes spp. & $\mathrm{ab}$ & $\mathrm{ab}$ & $\mathrm{ab}$ & $a b$ \\
\hline Amphora libyca EHRENB. & $\mathrm{ab}$ & & $\mathrm{ab}$ & \\
\hline Amphora montana KRASSKE & $\mathrm{ab}$ & & $\mathrm{ab}$ & $a b$ \\
\hline Amphora ovalis (KÜTZ.) KüTZ. & $\mathrm{ab}$ & & $\mathrm{ab}$ & $a b$ \\
\hline Amphora pediculus (KÜTZ.) GRUNOW & $\mathrm{ab}$ & $a b$ & $\mathrm{ab}$ & $a b$ \\
\hline Amphora veneta KÜTZ. & & & $\mathrm{ab}$ & \\
\hline Asterionella formosa HASSALL & $\mathrm{ab}$ & $a b$ & $\mathrm{ab}$ & $a b$ \\
\hline Caloneis amphisbaena (BORY) CLEVE & & $a b$ & $\mathrm{ab}$ & \\
\hline Caloneis bacillum (GRUNOW) CLEVE & $\mathrm{ab}$ & & & $a b$ \\
\hline Caloneis silicula (EHRENB.) Cleve & $\mathrm{ab}$ & & & \\
\hline Cocconeis pediculus EHRENB. & $\mathrm{ab}$ & $\mathrm{ab}$ & $\mathrm{ab}$ & \\
\hline Cocconeis placentula EHRENB. & $\mathrm{ab}$ & $a b$ & $\mathrm{ab}$ & \\
\hline Cymatopleura solea (BRÉB.) W. SM. var. solea & $\mathrm{ab}$ & $a b$ & $\mathrm{ab}$ & $a b$ \\
\hline Cymbella amphicephala NÄGELI & & $\mathrm{b}$ & & \\
\hline Cymbella cistula (EHRENB.) KIRCHN. & & & $\mathrm{ab}$ & \\
\hline Cymbela $\mathrm{cf}$. elginensis KRAMMER & & & $\mathrm{b}$ & \\
\hline
\end{tabular}




\begin{tabular}{|c|c|c|c|c|}
\hline Cont. Table 3 & $\mathbf{O L}$ & $\mathbf{L A}$ & DE & DY \\
\hline Cymbella helvetica KüTZ. & & $\mathrm{ab}$ & & \\
\hline Cymbella minuta HILSE ex RABENH. & $\mathrm{ab}$ & & $\mathrm{ab}$ & \\
\hline Cymbella silesiaca BLEISCH & $\mathrm{ab}$ & $\mathrm{ab}$ & $\mathrm{ab}$ & $a b$ \\
\hline Cymbella sinuata $\mathrm{W}$. GREG. & $\mathrm{ab}$ & $a b$ & $\mathrm{ab}$ & \\
\hline Cymbella tumida (BRÉB.) VAN HeURCK & & & $a b$ & \\
\hline Denticula tenuis KüTZ. & $\mathrm{ab}$ & & $a b$ & \\
\hline Diatoma anceps (EHRENB.) KIRCHN. & $\mathrm{b}$ & & & \\
\hline Diatoma ehrenbergii KÜTZ. & $\mathrm{ab}$ & & $\mathrm{ab}$ & $a b$ \\
\hline Diatoma mesodon (EHRENB.) KÜTZ. & $\mathrm{b}$ & $\mathrm{b}$ & $\mathrm{b}$ & \\
\hline Diatoma moniliformis KüTZ. & $a b$ & $\mathrm{ab}$ & $a b$ & $a b$ \\
\hline Diatoma tenuis C. AGARDH & & & $\mathrm{b}$ & \\
\hline Diatoma vulgaris BORY & $a b$ & $\mathrm{ab}$ & $\mathrm{ab}$ & $a b$ \\
\hline Didymosphenia geminata (LYNGBYE) M. SCHMIDT & $\mathrm{b}$ & & & \\
\hline Eunotia arcus EHRENB. & $\mathrm{b}$ & & & \\
\hline Eunotia incisa GREGORY & $a b$ & & & \\
\hline Eunotia exigua (BRÉB. ex KÜTZ.) RABENH. & $\mathrm{ab}$ & & & \\
\hline Eunotia praerupta EHRENB. & $\mathrm{ab}$ & & & \\
\hline Fragilaria arcus (EHRENB.) CLEVE & $\mathrm{ab}$ & $\mathrm{ab}$ & $a b$ & \\
\hline Fragilaria bicapitata MAYER & $\mathrm{b}$ & $\mathrm{b}$ & & \\
\hline Fragilaria brevistriata GRUNOW & & & $\mathrm{ab}$ & \\
\hline $\begin{array}{l}\text { Fragilaria capucina DESM. var. capitellata (GRUNOW) LANGE- } \\
\text { BERT. }\end{array}$ & $\mathrm{b}$ & & & \\
\hline Fragilaria capucina var. perminuta (GRUNOW) LANGE-BERT. & $\mathrm{b}$ & & & \\
\hline Fragilaria capucina var. vaucheriae (KÜTZ.) LANGE-BERT. & $\mathrm{ab}$ & $a b$ & $\mathrm{ab}$ & $a b$ \\
\hline Fragilaria construens (EHRENB.) GRUNOW & & & & $\mathrm{ab}$ \\
\hline Fragilaria crotonensis KITTON & $\mathrm{ab}$ & & $\mathrm{ab}$ & $\mathrm{ab}$ \\
\hline Fragilaria elliptica SCHUM. & & $\mathrm{b}$ & $\mathrm{b}$ & \\
\hline Fragilaria leptostauron (EHRENB.) HUST. & $\mathrm{ab}$ & & & \\
\hline Fragilaria nanana LANGE.-BERT. & & & $\mathrm{b}$ & \\
\hline Fragilaria nitzschioides GRUNOW & & & $\mathrm{b}$ & \\
\hline Fragilaria parasitica (W. SM.) GRUNOW var. parasitica & $\mathrm{ab}$ & & & \\
\hline Fragilaria parasitica var. subconstricta GRUNOW & $\mathrm{ab}$ & & $a b$ & \\
\hline Fragilaria pinnata EHRENB. & $\mathrm{ab}$ & & $\mathrm{ab}$ & \\
\hline Fragilaria ulna var. acus (KÜTZ.) LANGE-BERT. & $\mathrm{ab}$ & $a b$ & $\mathrm{ab}$ & \\
\hline Fragilaria ulna (NITZSCH) LANGE-BERT. var. ulna & $\mathrm{ab}$ & $a b$ & $a b$ & $\mathrm{ab}$ \\
\hline Frustulia vulgaris (THWAITES) DE TONI & & & $a b$ & \\
\hline Gomphonema acuminatum EHRENB. & $\mathrm{b}$ & & & \\
\hline Gomphonema affine KüTZ. & & & $\mathrm{ab}$ & \\
\hline Gomphonema angustatum (KÜTZ.) RABENH. & $\mathrm{ab}$ & & $a b$ & \\
\hline Gomphonema angustum C. AGARDH & $\mathrm{ab}$ & & & \\
\hline Gomphonema clavatum EHRENB. & $\mathrm{ab}$ & & & \\
\hline Gomphonema gracile EHRENB. & & & $a b$ & \\
\hline Gomphonema insigne GREGORY & & & $\mathrm{b}$ & \\
\hline Gomphonema olivaceum (HORNEM.) BRÉB. & $\mathrm{ab}$ & $\mathrm{ab}$ & $\mathrm{ab}$ & $a b$ \\
\hline Gomphonema parvulum (KüTZ.) KüTZ. & $\mathrm{ab}$ & $\mathrm{ab}$ & $\mathrm{ab}$ & $a b$ \\
\hline Gomphonema pumilum (GRUNOw) REICHARDT et LANGE-BERT. & $\mathrm{b}$ & $\mathrm{b}$ & $\mathrm{b}$ & \\
\hline Gomphonema tergestinum (GRUNOW) FRICKE & $\mathrm{b}$ & $\mathrm{b}$ & $\mathrm{b}$ & \\
\hline Gomphonema truncatum EHRENB. & $\mathrm{ab}$ & & & \\
\hline Gomphonema sp. & $\mathrm{ab}$ & $a b$ & & \\
\hline Gyrosigma acuminatum (KÜTZ.) RABENH. & $\mathrm{ab}$ & $a b$ & $\mathrm{ab}$ & $a b$ \\
\hline
\end{tabular}




\begin{tabular}{|c|c|c|c|c|}
\hline Cont. Table 3 & $\mathbf{O L}$ & $\mathbf{L A}$ & DE & $\mathbf{D Y}$ \\
\hline Gyrosigma attenuatum (KÜTZ.) RABENH. & & & $a b$ & $\mathrm{ab}$ \\
\hline Gyrosigma scalproides (RABENH.) CLEVE & $\mathrm{ab}$ & & $a b$ & $? \mathrm{ab}$ \\
\hline Hantzschia amphioxys (EHRENB.) GRUNOW & $a b$ & $\mathrm{ab}$ & $a b$ & $\mathrm{ab}$ \\
\hline Meridion circulare (GREVILLE) C. AGARDH var. circulare & $\mathrm{ab}$ & $\mathrm{ab}$ & $\mathrm{ab}$ & \\
\hline Meridion circulare var. constrictum (RALFS) VAN HEURCK & $\mathrm{ab}$ & $\mathrm{ab}$ & & \\
\hline Navicula atomus (KÜTZ.) GRUNOW & a & & & \\
\hline Navicula bacillum EHRENB. & a & & a & \\
\hline Navicula capitata EHRENB. & $\mathrm{ab}$ & $\mathrm{ab}$ & $\mathrm{ab}$ & $\mathrm{ab}$ \\
\hline Navicula capitatoradiata H. GERM. & $a b$ & $\mathrm{ab}$ & $a b$ & $a b$ \\
\hline Navicula cincta (EHRENB.) RALFS & & & $a b$ & \\
\hline Navicula citrus KRASSKE & & & $a b$ & $\mathrm{ab}$ \\
\hline Navicula confervacea (KüTZ.) GRUNOW & & $\mathrm{b}$ & $\mathrm{b}$ & \\
\hline Navicula contenta GRUNOW & $\mathrm{b}$ & & & \\
\hline Navicula cryptocephala KüTZ. & & & $\mathrm{b}$ & \\
\hline Navicula cryptotenella LANGE.-BERT. & $\mathrm{b}$ & & $\mathrm{b}$ & \\
\hline Navicula cuspidata (KüTZ.) KÜTZ. & & & $a b$ & \\
\hline Navicula decussis OESTRUP & $a b$ & & & \\
\hline Navicula elginensis (GREGORY) RALFS & $\mathrm{b}$ & & & \\
\hline Navicula erifuga LANGE-BERT. & & & $\mathrm{b}$ & \\
\hline Navicula goeppertiana (BLEISCH) H.L. SM. & a & & & \\
\hline Navicula gregaria DONKIN & $a b$ & & $\mathrm{ab}$ & $\mathrm{ab}$ \\
\hline Navicula halophila (GRUNOw) CLEVE & & a & & \\
\hline Navicula helensis P. SCHULZ & $\mathrm{b}$ & & & \\
\hline Navicula lanceolata (C. AGARDH) EHRENB. & $a b$ & $a b$ & $a b$ & $\mathrm{ab}$ \\
\hline Navicula medioconvexa Hust. & $\mathrm{b}$ & & & \\
\hline Navicula menisculus ScHUM. & $a b$ & $a b$ & $a b$ & $\mathrm{ab}$ \\
\hline Navicula mutica KüTZ. & $a b$ & & & \\
\hline Navicula nivalis EHRENB. & a & a & & \\
\hline Navicula placentula (EHRENB.) GRUNOW & & & $a b$ & \\
\hline Navicula protracta (GRUNOW) CLEVE & $a b$ & & & \\
\hline Navicula pupula KÜTZ. & $a b$ & & $a b$ & $\mathrm{ab}$ \\
\hline Navicula pygmaea KüTZ. & $a b$ & & $a b$ & \\
\hline Navicula radiosa KüTZ. & $\mathrm{b}$ & & & \\
\hline Navicula slesvicensis KüTZ. & $\mathrm{ab}$ & & $\mathrm{ab}$ & \\
\hline Navicula subminuscula MANGUIN & $a b$ & $\mathrm{ab}$ & $a b$ & $\mathrm{ab}$ \\
\hline Navicula subplacentula Hust. & & & $\mathrm{b}$ & \\
\hline Navicula tripunctata (O.F. MÜLL.) BORY & $a b$ & $\mathrm{ab}$ & $a b$ & \\
\hline Navicula trivialis LANGE-BERT. & $a b$ & & $a b$ & $\mathrm{ab}$ \\
\hline Navicula veneta KüTZ. & $a b$ & & & $a b$ \\
\hline Navicula viridula (KüTZ.) EHRENB. var. linearis Hust. & $a b$ & $\mathrm{ab}$ & $a b$ & \\
\hline Navicula viridula var. rostellata (KüTZ.) CLEVE & & & & $\mathrm{ab}$ \\
\hline Nitzschia acicularis (KüTZ.) W. SM. & & $\mathrm{ab}$ & $\mathrm{ab}$ & \\
\hline Nitzschia acicularioides Hust. & $\mathrm{a}$ & & & \\
\hline Nitzschia amphibia GRUNOW & $\mathrm{ab}$ & & $\mathrm{ab}$ & \\
\hline Nitzschia angustata (W. SM.) GRUNOW & $a b$ & & & \\
\hline Nitzschia calida GRUNOW & & & $\mathrm{ab}$ & \\
\hline Nitzschia capitellata Hust. & $a b$ & $\mathrm{ab}$ & $a b$ & $a b$ \\
\hline Nitzschia constricta (KÜTZ.) RALFS & $a b$ & $\mathrm{ab}$ & $\mathrm{ab}$ & $a b$ \\
\hline Nitzschia dissipata (KüTZ.) GRUNOW & $a b$ & $\mathrm{ab}$ & & \\
\hline
\end{tabular}




\begin{tabular}{|c|c|c|c|c|}
\hline Cont. Table 3 & $\mathbf{O L}$ & $\mathbf{L A}$ & $\mathbf{D E}$ & DY \\
\hline Nitzschia dubia W. SM. & $\mathrm{b}$ & & $\mathrm{b}$ & \\
\hline Nitzschia flexa Scнuм. & & & $\mathrm{b}$ & \\
\hline Nitzschia fonticola GRUNOW & a & a & a & $\mathrm{a}$ \\
\hline Nitzschia frustulum (KÜTZ.) GRUNOW & a & $\mathrm{a}$ & a & $\mathrm{a}$ \\
\hline Nitzschia fruticosa Hust. & $\mathrm{ab}$ & $\mathrm{ab}$ & $\mathrm{ab}$ & \\
\hline Nitzschia gracilis HANTZSCH & & & a & $\mathrm{a}$ \\
\hline Nitzschia graciliformis LANGE-BERT. et SIMONSEN & $\mathrm{ab}$ & $a b$ & $\mathrm{ab}$ & \\
\hline Nitzschia hantzschiana RABENH. & $\mathrm{ab}$ & & & \\
\hline Nitzschia heufleriana GRUNOw & $\mathrm{ab}$ & & $\mathrm{ab}$ & $a b$ \\
\hline Nitzschia hungarica GRUNOW & $\mathrm{ab}$ & $\mathrm{ab}$ & $\mathrm{ab}$ & $a b$ \\
\hline Nitzschia inconspicua GRUNOW & $\mathrm{ab}$ & & $\mathrm{ab}$ & $\mathrm{ab}$ \\
\hline Nitzschia levidensis (W. SM.) GRUNOW & $\mathrm{ab}$ & $\mathrm{ab}$ & $a b$ & $\mathrm{ab}$ \\
\hline Nitzschia liebetruthii RABENH. & & & $\mathrm{ab}$ & \\
\hline Nitzschia linearis (C. AGARDH) W. SM. & $\mathrm{ab}$ & $\mathrm{ab}$ & $a b$ & $\mathrm{ab}$ \\
\hline Nitzschia littoralis GRUNOW & a & a & & \\
\hline Nitzschia palea (KüTZ.) W. SM. & & $\mathrm{ab}$ & $\mathrm{ab}$ & \\
\hline Nitzschia pusilla GRUNOW & a & & & \\
\hline Nitzschia recta HANTZSCH & $\mathrm{ab}$ & & $\mathrm{ab}$ & \\
\hline Nitzschia sigma (KÜTZ.) W. SM. & a & & & \\
\hline Nitzschia sigmoidea (NITZSCH) W. SM. & $\mathrm{ab}$ & & & \\
\hline Nitzschia sinuata var. delognei (GRUNOW) LANGE.-BERT. & & & a & \\
\hline Nitzschia sinuata var. tabellaria (GRUNOw) GRUNOw & a & & & \\
\hline Nitzschia sociabilis Hust. & & & a & \\
\hline Nitzschia subacicularis HusT. & $\mathrm{ab}$ & $a b$ & $\mathrm{ab}$ & \\
\hline Nitzschia tubicola GRUNOW & $\mathrm{ab}$ & & $a b$ & \\
\hline Nitzschia umbonata (EHRENB.) LANGE-BERT. & $\mathrm{ab}$ & $\mathrm{ab}$ & & \\
\hline Nitzschia vermicularis (KÜTZ.) HANTZSCH & a & & & \\
\hline Nitzschia spp. & $\mathrm{ab}$ & $\mathrm{ab}$ & $\mathrm{ab}$ & $a b$ \\
\hline Pinnularia borealis EHRENB. & a & & & \\
\hline Pinnularia lundii Hust. & a & & & \\
\hline Pinnularia microstauron (EHRENB.) A.CleVE & & $\mathrm{ab}$ & $\mathrm{ab}$ & \\
\hline Pinnularia obscura KRASSKE & & a & & \\
\hline Pinnularia subcapitata $\mathrm{W}$. GREG. & a & a & & \\
\hline Pinnularia subrostrata (A.ClEVE) CLEVE-EULER & & & $\mathrm{b}$ & \\
\hline Pinnularia sp. & $\mathrm{ab}$ & $\mathrm{ab}$ & $\mathrm{ab}$ & \\
\hline Rhoicosphenia abbreviata (C. AGARDH) LANGE-BERT. & $\mathrm{ab}$ & $\mathrm{ab}$ & $\mathrm{ab}$ & $\mathrm{ab}$ \\
\hline Stauroneis phoenicenteron (NITZSCH) EHRENB. & $\mathrm{ab}$ & & & \\
\hline Stauroneis smithii GRUNOW & a & & & \\
\hline Surirella angusta KÜTZ. & $\mathrm{ab}$ & & & \\
\hline Surirella bifrons EHRENB. & & & $\mathrm{b}$ & \\
\hline Surirella brebissonii var. kuetzingii KRAMMER et LANGE-BERT. & & $\mathrm{ab}$ & $\mathrm{ab}$ & $\mathrm{ab}$ \\
\hline Surirella capronii BRÉB. & & & $\mathrm{b}$ & \\
\hline Surirella crumena BRÉB. & $\mathrm{ab}$ & & & \\
\hline Surirella linearis W. SM. var. linearis & & $\mathrm{ab}$ & & \\
\hline Surirella linearis var. helvetica (BRUN.) F. MESITER & & & & $\mathrm{a}$ \\
\hline Surirella minuta BRÉB. & $\mathrm{ab}$ & $\mathrm{ab}$ & & \\
\hline Surirella ovalis BRÉB. & a & & & \\
\hline Surirella tenera $\mathrm{W}$. GREG. & $\mathrm{ab}$ & & $\mathrm{ab}$ & $\mathrm{ab}$ \\
\hline Surirella terricola LANGE-BERT. et ALLES & & $\mathrm{ab}$ & $\mathrm{ab}$ & \\
\hline Surirella spp. & $a b$ & & $a b$ & \\
\hline
\end{tabular}




\begin{tabular}{|c|c|c|c|c|}
\hline Cont. Table 3 & OL & $\mathbf{L A}$ & $\mathbf{D E}$ & DY \\
\hline Tabellaria flocculosa (RоTH) KüTZ. & $\mathrm{a}$ & & & \\
\hline \multicolumn{5}{|l|}{ CRYPTOPHYCEAE } \\
\hline Chroomonas nordstedtii HANSG. & $\mathrm{ab}$ & $\mathrm{ab}$ & $\mathrm{ab}$ & \\
\hline Cryptomonas compressa PASCHER & $\mathrm{ab}$ & & & \\
\hline Cryptomonas ovata EHRENB. & $\mathrm{ab}$ & $\mathrm{ab}$ & $a b$ & \\
\hline Cryptomonas spp. & $\mathrm{ab}$ & $\mathrm{ab}$ & $a b$ & $\mathrm{ab}$ \\
\hline Rhodomonas lacustris PASCHER et RUTTNER & a & & & \\
\hline \multicolumn{5}{|l|}{ DINOPHYCEAE } \\
\hline Ceratium hirundinella (O. F. Müll.) Bergh & $\mathrm{ab}$ & $a b$ & $a b$ & $a b$ \\
\hline Gymnodinium spp. & $\mathrm{ab}$ & $\mathrm{ab}$ & $a b$ & $a b$ \\
\hline Peridinium spp. & $\mathrm{ab}$ & $\mathrm{ab}$ & $\mathrm{ab}$ & $a b$ \\
\hline \multicolumn{5}{|l|}{ XANTHOPHYCEAE } \\
\hline Centritractus belenophorus LEMMERM. & & & $a b$ & \\
\hline Goniochloris fallax FOTT & $\mathrm{ab}$ & $\mathrm{ab}$ & $\mathrm{ab}$ & $a b$ \\
\hline Goniochloris mutica (A.K.H. BRAUN) FOTT & $\mathrm{ab}$ & $\mathrm{ab}$ & $\mathrm{ab}$ & $\mathrm{ab}$ \\
\hline Goniochloris sp. & & & $a b$ & \\
\hline Ophiocytium capitatum WOLLE & & & $a b$ & \\
\hline Pseudostaurastrum hastatum (REINSCH) CHODAT & a & a & $a b$ & a \\
\hline Tetraedriella jovetii (BOURR.) BOURR. & & & $\mathrm{b}$ & \\
\hline Pseudotetraedron neglectum PASCHER & & & $a b$ & \\
\hline \multicolumn{5}{|l|}{ CHLOROPHYCEAE } \\
\hline \multicolumn{5}{|l|}{ Volvocales } \\
\hline Carteria globosa KORSHIKOV ex PASCHER & & & $a b$ & \\
\hline Carteria multifilis (FRESEN.) O. DILL & & & $\mathrm{b}$ & \\
\hline Carteria radiosa KORSHIKOV ex PASCHER & $\mathrm{ab}$ & $\mathrm{ab}$ & $a b$ & $a b$ \\
\hline Carteria sp. & a & a & $?$ & $\mathrm{a}$ \\
\hline Chlamydomonas bicocca PASCHER & & & a & \\
\hline Chlamydomonas bilatus H. ETTL & & a & & \\
\hline Chlamydomonas debaryana GOROZH. & $\mathrm{b}$ & & & \\
\hline Chlamydomonas gloeophila SKUJA & & & $\mathrm{ab}$ & \\
\hline Chlamydomonas incerta PASCHER & a & & & \\
\hline Chlamydomonas monadina F. STEIN & $\mathrm{ab}$ & $\mathrm{ab}$ & $\mathrm{ab}$ & $\mathrm{ab}$ \\
\hline Chlamydomonas pertusa CHODAT & & & a & \\
\hline Chlamydomonas pseudolunata $\mathrm{H}$. ЕтTL & & & $\mathrm{ab}$ & \\
\hline Chlamydomonas pseudopertusa $\mathrm{H}$. ЕTтL & & $\mathrm{ab}$ & $a b$ & \\
\hline Chlamydomonas reinhardtii P.A. DANG. & a & a & a & a \\
\hline Chlamydomonas simplex PASCHER & $\mathrm{ab}$ & $\mathrm{ab}$ & $\mathrm{ab}$ & \\
\hline Chlamydomonas skujae PASCHER & & & a & \\
\hline Chlorogonium elongatum P. A. DANG. & & $\mathrm{ab}$ & $\mathrm{ab}$ & \\
\hline Chlorogonium fusiforme MATV. & & & $\mathrm{ab}$ & \\
\hline Chlorogonium minimum PLAYFAIR & a & & & \\
\hline Chloromonas anurae (KORSHIKOV) GERLOFF et H. ETTL & & a & & \\
\hline Eudorina elegans EHRENB. & a & a & a & \\
\hline Eudorina unicocca G. M. SмIтH & $\mathrm{b}$ & $\mathrm{b}$ & $\mathrm{b}$ & $\mathrm{b}$ \\
\hline Gloeomonas diplochlamys (SKUJA) H. ЕTTL & a & $\mathrm{a}$ & $\mathrm{a}$ & $\mathrm{a}$ \\
\hline Gloeomonas lateperforata (SKUנA) H. ЕTтL & & $\mathrm{a}$ & & \\
\hline Gloeomonas tecta (SKUJA) H. et O. ЕTTL & & $\mathrm{ab}$ & & \\
\hline Gonium pectorale O. F. MüLL. & & $\mathrm{ab}$ & $a b$ & \\
\hline Gonium sociale (DUJARD) WARM. & & & a & \\
\hline Haematococcus buetschlii ВцОснм. & & & $\mathrm{a}$ & \\
\hline
\end{tabular}




\begin{tabular}{|c|c|c|c|c|}
\hline Cont. Table 3 & $\mathbf{O L}$ & $\overline{\mathbf{L A}}$ & $\overline{\mathrm{DE}}$ & DY \\
\hline Lobomonas ampla PASCHER & $\mathrm{ab}$ & $\mathrm{ab}$ & & \\
\hline Nephroselmis olivacea F. STEIN & & & $a b$ & \\
\hline Pandorina morum (O. F. MÜLL.) BORY & $a b$ & $a b$ & $\mathrm{ab}$ & $a b$ \\
\hline Phacotus lenticularis (EHRENB.) F. STEIN & $a b$ & $\mathrm{ab}$ & $a b$ & $a b$ \\
\hline Pleudorina californica W. SHAW & & & $\mathrm{ab}$ & $a b$ \\
\hline Pseudocarteria peterhoffiensis (KISSELEV) H. ETTL & a & a & a & \\
\hline Pteromonas aculeata LEMMERM. & $\mathrm{b}$ & $\mathrm{b}$ & $\mathrm{b}$ & \\
\hline Pteromonas angulosa (H. J. CARTER) LEMMERM. & a & $\mathrm{a}$ & a & $\mathrm{a}$ \\
\hline Pteromonas cordiformis LEMMERM. & $a b$ & $a b$ & $a b$ & \\
\hline Pteromonas limnetica Новтов. & & & a & \\
\hline Sphaerellopsis aulata (PASCHER) GERLOFF & & & $a b$ & $a b$ \\
\hline Sphaerellopsis gloeosphaera (PASCHER et JAHODA) H. et O. ETTL & & & & $\mathrm{b}$ \\
\hline Tetraselmis cordiformis (H. J. CARTER) F. STEIN & & $\mathrm{ab}$ & $\mathrm{ab}$ & \\
\hline \multicolumn{5}{|l|}{ Tetrasporales } \\
\hline Chlorangiopsis flos-aquae HINDÁK et HINDÁKOVÁ & & $\mathrm{b}$ & $\mathrm{b}$ & $\mathrm{b}$ \\
\hline Pseudosphaerocystis lacustris (LEMMERM.) NOVÁKOVÁ & & a & & \\
\hline Stylosphaeridium stipitatum (H. BACHM.) GEITLER et GIMESI & & & $\mathrm{b}$ & \\
\hline \multicolumn{5}{|l|}{ Chlorococcales } \\
\hline Acanthosphaera zachariasii LEMMERM. & $a b$ & $a b$ & $\mathrm{ab}$ & $a b$ \\
\hline Actinastrum hantzschii LAGERH. & $a b$ & $\mathrm{ab}$ & $\mathrm{ab}$ & $a b$ \\
\hline Amphikrikos nanus (FOTT et HEYNIG) HINDÁK & & $\mathrm{b}$ & & \\
\hline Ankistrodesmus falcatus (CORDA) RALFS & & a & a & $\mathrm{a}$ \\
\hline Ankistrodesmus fusiformis CORDA & & & a & \\
\hline Ankyra ancora f. issajevii (KISSELEV) FOTT & & & $\mathrm{b}$ & $\mathrm{b}$ \\
\hline Botryococcus braunii KüTZ. & & & $a b$ & $a b$ \\
\hline Chlorella vulgaris BEIJ. & $a b$ & $a b$ & $\mathrm{ab}$ & $a b$ \\
\hline Closteriopsis acicularis (G.M. SMITH) J.H. BELCHER et SwALE & a & & & \\
\hline Closteriopsis longissima (LEMMERM.) LEMMERM. & & $a b$ & & \\
\hline Coelastrum astroideum DE NoT. & $a b$ & $a b$ & $a b$ & $a b$ \\
\hline Coelastrum microporum NÄGELI & $a b$ & $a b$ & $a b$ & $a b$ \\
\hline Coelastrum polychordum (KORSHIKOV) HINDÁK & & & $\mathrm{b}$ & \\
\hline Coelastrum reticulatum (P.A. DANG.) SENN & a & a & & $\mathrm{a}$ \\
\hline Coenochloris astroidea HINDÁK & a & & & $\mathrm{a}$ \\
\hline Coenochloris planctonica (KORSHIKOV) HINDÁK & $a b$ & $a b$ & $a b$ & $a b$ \\
\hline Coenochloris polycocca (KORSHIKOV) HINDÁK & $\mathrm{ab}$ & $a b$ & $a b$ & $a b$ \\
\hline Coenococcus planctonicus KORSHIKOV & $a b$ & $a b$ & $a b$ & $\mathrm{ab}$ \\
\hline Coenocystis subcylindrica KORSHIKOV & $\mathrm{b}$ & & $\mathrm{b}$ & \\
\hline Crucigenia tetrapedia (KIRCHN.) W. et G. S. WEST & $\mathrm{ab}$ & $a b$ & $a b$ & $a b$ \\
\hline Crucigeniella apiculata (LEMMERM.) KOMÁREK & $a b$ & $a b$ & $a b$ & $a b$ \\
\hline Diacanthos belenophorus KORSHIKOV & & $a b$ & $a b$ & \\
\hline Dicellula geminata (PRINTZ) KORSHIKOV & & & $\mathrm{b}$ & $\mathrm{b}$ \\
\hline Dichotomococcus curvatus KORSHIKOV & & $\mathrm{a}$ & a & \\
\hline Dictyosphaerium pulchellum H.C. WoOD & & & a & \\
\hline Dictyosphaerium tetrachotomum PRINTZ & $a b$ & $a b$ & $a b$ & $a b$ \\
\hline Didymocystis inermis (FоTт) FоTт & & $a b$ & $a b$ & \\
\hline Didymogenes anomala(G.M. SMITH) HINDÁK & & $\mathrm{b}$ & $\mathrm{b}$ & \\
\hline Didymogenes palatina SCHMIDLE & & & $\mathrm{b}$ & \\
\hline Dimorphococcus lunatus A.K.H. BRAUN & & & $\mathrm{b}$ & \\
\hline Diplochloris lunata (Fотт) Fотт & & & a & \\
\hline Granulocystis helenae HINDÁK & & $\mathrm{b}$ & $\mathrm{b}$ & \\
\hline
\end{tabular}




\begin{tabular}{|c|c|c|c|c|}
\hline Cont. Table 3 & $\mathbf{O L}$ & $\mathbf{L A}$ & DE & DY \\
\hline Granulocystopsis coronata (LEMMERM.) HINDÁK & $\mathrm{b}$ & $\mathrm{b}$ & $\mathrm{b}$ & \\
\hline Granulocystopsis elegans (FOTT) HINDÁK & $\mathrm{b}$ & $\mathrm{b}$ & & \\
\hline Franceia echidna (BOHLIN) BOURR. & $\mathrm{ab}$ & $\mathrm{ab}$ & $\mathrm{ab}$ & $a b$ \\
\hline Franceia tenuispina KORSHIKOV & $\mathrm{ab}$ & $\mathrm{ab}$ & $\mathrm{ab}$ & $a b$ \\
\hline Juraniella javorkae (НовтOв.) НОRTOВ. & $\mathrm{ab}$ & & & \\
\hline Kirchneriella aperta TEILING & & $\mathrm{b}$ & & \\
\hline Kirchneriella incurvata BLECH. et SWALE & & $\mathrm{ab}$ & & \\
\hline Kirchneriella obesa (W. WEST) SCHMIDLE & a & a & a & $\mathrm{a}$ \\
\hline Lagerheimia ciliata (LAGERH.) CHODAT & $\mathrm{ab}$ & $\mathrm{ab}$ & $\mathrm{ab}$ & $a b$ \\
\hline Lagerheimia genevenis (CHODAT) СНОDAT & $\mathrm{ab}$ & $\mathrm{ab}$ & $\mathrm{ab}$ & \\
\hline Lagerheimia longiseta (LEMMERM.) WILLE & & $\mathrm{b}$ & $\mathrm{b}$ & \\
\hline Lagerheimia marssonii LEMMERM. & & & $\mathrm{b}$ & \\
\hline Lagerheimia quadriseta (LEMMERM.) G. M. SMITH & & & a & \\
\hline Lagerheimia subsalsa LEMMERM. & & $\mathrm{b}$ & & \\
\hline Lagerheimia wratislawiensis SCHRÖDER & $\mathrm{b}$ & & $\mathrm{b}$ & \\
\hline Micractinium bornhemiense (CONR.) KORSHIKOV & $\mathrm{ab}$ & $a b$ & $a b$ & \\
\hline Micractinium crassisetum Новтов. & & & $?$ & a \\
\hline Micractinium pusillum FRESEN. & $\mathrm{ab}$ & $\mathrm{ab}$ & $\mathrm{ab}$ & $a b$ \\
\hline Micractinium quadrisetum (LEMMERM.) G. M. SMITH & & $a b$ & $a b$ & \\
\hline Monoraphidium arcuatum (KORSHIKOV) HINDÁK & $\mathrm{ab}$ & $\mathrm{ab}$ & $\mathrm{ab}$ & \\
\hline Monoraphidium contortum (THUR.) KOMÁRK.-LEGN. & $\mathrm{ab}$ & $a b$ & $a b$ & $\mathrm{ab}$ \\
\hline Monoraphidium convolutum (CORDA) KOMÁRK.-LEGN. & & & $a b$ & \\
\hline Monoraphidium intermedium HINDÁK & $a b$ & $\mathrm{ab}$ & $\mathrm{ab}$ & $a b$ \\
\hline Monoraphidium griffithii (BERKEL.) KOMÁRK.-LEGN. & $\mathrm{ab}$ & $a b$ & $\mathrm{ab}$ & \\
\hline Monoraphidium pseudobraunii (J. H. BELCHER et SwALE) HEYNIG & & & $\mathrm{ab}$ & \\
\hline Neocystis diplococca (HINDÁK) HINDÁK & & & $a b$ & \\
\hline Neodesmus danubialis HINDÁK & & & $\mathrm{b}$ & \\
\hline Nephrochlamys rotunda KORSHIKOV & $\mathrm{b}$ & & & \\
\hline Nephrochlamys subsolitaria (G. S. WEST) KORSHIKOV & $\mathrm{ab}$ & $a b$ & $a b$ & \\
\hline Nephrochlamys willeana (PRINTZ) KORSHIKOV & $\mathrm{b}$ & & & \\
\hline Nephrocytium agardhianum NÄGELI & a & & a & \\
\hline Oocystella borgei (J. SNOw) HINDÁK & $\mathrm{ab}$ & $\mathrm{ab}$ & $\mathrm{ab}$ & \\
\hline Oocystella lacustris (СHODAT) HINDÁK & $\mathrm{ab}$ & $\mathrm{ab}$ & $\mathrm{ab}$ & $\mathrm{ab}$ \\
\hline Oocystella marssonii (LEMMERM.) HINDÁK & $\mathrm{ab}$ & $\mathrm{ab}$ & $a b$ & $a b$ \\
\hline Paradoxia multiseta SVIRENKO & & & & $\mathrm{b}$ \\
\hline Pediastrum biradiatum MEYEN & a & & a & \\
\hline Pediastrum boryanum (TURPIN) MENEGH. & $\mathrm{ab}$ & $a b$ & $a b$ & $a b$ \\
\hline Pediastrum duplex MEYEN var. duplex & $\mathrm{ab}$ & $a b$ & $\mathrm{ab}$ & $a b$ \\
\hline Pediastrum duplex var. gracillimum W. et G. S. WEST & a & a & a & a \\
\hline Pediastrum simplex MEYEN & $\mathrm{ab}$ & $\mathrm{ab}$ & $\mathrm{ab}$ & $\mathrm{ab}$ \\
\hline Pediastrum tetras (EHRENB.) RALFS & $\mathrm{ab}$ & $\mathrm{ab}$ & $\mathrm{ab}$ & $a b$ \\
\hline Planktosphaeria gelatinosa G. M. SмIтH & $\mathrm{ab}$ & $\mathrm{ab}$ & $\mathrm{ab}$ & $a b$ \\
\hline Polyedriopsis spinulosa (SCHMIDLE) SCHMIDLE & $\mathrm{ab}$ & & $\mathrm{ab}$ & $a b$ \\
\hline Pseudodictyosphaerium jurisii (HINDÁK) HINDÁK & $\mathrm{ab}$ & $\mathrm{ab}$ & $a b$ & $a b$ \\
\hline Pseudodictyosphaerium minusculum HINDÁK & $\mathrm{ab}$ & $\mathrm{ab}$ & $\mathrm{ab}$ & \\
\hline Pseudodidymocystis inconspicua (KORSHIKOV) HINDÁK & $\mathrm{ab}$ & & $\mathrm{ab}$ & \\
\hline $\begin{array}{l}\text { Pseudodidymocystis planctonica (KORSHIKOV) E.H. HEGEW. et } \\
\text { DEASON }\end{array}$ & & & $\mathrm{b}$ & $\mathrm{b}$ \\
\hline Pseudokirchneriella contorta (SCHMIDLE) HINDÁK & $\mathrm{ab}$ & $\mathrm{ab}$ & $\mathrm{ab}$ & \\
\hline Pseudokirchneriella danubiana (HINDÁK) HINDÁK & & & $\mathrm{ab}$ & \\
\hline Pseudokirchneriella irregularis (G. M. SMITH) HINDÁK & & & a & \\
\hline
\end{tabular}




\begin{tabular}{|c|c|c|c|c|}
\hline Cont. Table 3 & $\mathbf{O L}$ & $\mathbf{L A}$ & $\mathbf{D E}$ & DY \\
\hline Pseudokirchneriella roselata (HINDÁK) HINDÁK & & $\mathrm{ab}$ & & \\
\hline Pseudotetrastrum punctatum (SCHMIDLE) HINDÁK & $\mathrm{b}$ & & $\mathrm{b}$ & \\
\hline Quadricoccus laevis Fотт & a & a & & $\mathrm{a}$ \\
\hline Quadricoccus verrucosus FОTT & & $\mathrm{b}$ & $\mathrm{b}$ & \\
\hline Radiococcus planctonicus J. W. G. LuND & & & a & \\
\hline Raphidocelis sigmoidea HINDÁK & & & $a b$ & \\
\hline Scenedesmus abundans (KIRCHN.) CHODAT & $\mathrm{ab}$ & $\mathrm{ab}$ & $a b$ & $a b$ \\
\hline Scenedesmus acuminatus (LAGERH.) CHODAT & $\mathrm{ab}$ & $\mathrm{ab}$ & $a b$ & $\mathrm{ab}$ \\
\hline Scenedesmus arcuatus (LEMMERM.) LEMMERM. & a & $\mathrm{a}$ & a & a \\
\hline Scenedesmus armatus (СHODAT) CHODAT var. armatus & $\mathrm{ab}$ & $\mathrm{ab}$ & $a b$ & $a b$ \\
\hline Scenedesmus armatus var. bicaudatus (GUGLIELM.) СНОDAT & $\mathrm{ab}$ & $\mathrm{ab}$ & $a b$ & $\mathrm{ab}$ \\
\hline Scenedesmus bernardii G. M. SмIтH & $\mathrm{ab}$ & $\mathrm{ab}$ & $a b$ & $\mathrm{ab}$ \\
\hline Scenedesmus brasiliensis BoHLIN & & a & a & \\
\hline Scenedesmus carinatus (LEMMERM.) CHODAT & & $\mathrm{ab}$ & $\mathrm{ab}$ & \\
\hline Scenedesmus communis E. H. HEGEW. & $\mathrm{ab}$ & $\mathrm{ab}$ & $a b$ & $\mathrm{ab}$ \\
\hline Scenedesmus denticulatus LAGERH. var. denticulatus & $\mathrm{ab}$ & $\mathrm{ab}$ & $a b$ & $a b$ \\
\hline Scenedesmus denticulatus var. linearis HANSG. & $\mathrm{ab}$ & $\mathrm{ab}$ & $\mathrm{ab}$ & $\mathrm{ab}$ \\
\hline Scenedesmus dispar BRÉB. & $\mathrm{ab}$ & $\mathrm{ab}$ & $a b$ & $\mathrm{ab}$ \\
\hline Scenedesmus ellipticus CORDA & & $\mathrm{b}$ & $\mathrm{b}$ & \\
\hline Scenedesmus intermedius CHODAT & $\mathrm{ab}$ & & $a b$ & \\
\hline Scenedesmus longispina СНОDАТ & $\mathrm{ab}$ & & $a b$ & \\
\hline Scenedesmus maximus (W. et G. S. WEST) CHODAT & $\mathrm{ab}$ & $a b$ & $a b$ & $a b$ \\
\hline Scenedesmus obliquus (TURPIN) KüTZ. & a & a & a & $\mathrm{a}$ \\
\hline Scenedesmus obtusus MEYEN & a & a & & \\
\hline Scenedesmus opoliensis P. G. RichT. & a & a & a & \\
\hline Scenedesmus pannonicus Новтов. & $\mathrm{ab}$ & $\mathrm{ab}$ & $a b$ & $a b$ \\
\hline Scenedesmus pectinatus MEYEN & a & a & a & \\
\hline Scenedesmus raciborskii WoŁosz. & $\mathrm{ab}$ & $\mathrm{ab}$ & $a b$ & $a b$ \\
\hline Scenedesmus serratus (CORDA) BOHLIN & & $\mathrm{ab}$ & $a b$ & \\
\hline Scenedesmus subspicatus CHODAT & $\mathrm{ab}$ & $\mathrm{ab}$ & $a b$ & $a b$ \\
\hline Scenedesmus verrucosus Y. V. RoLL & $\mathrm{ab}$ & $\mathrm{ab}$ & $\mathrm{ab}$ & \\
\hline Schroederia robusta KORSHIKOV & & $\mathrm{b}$ & $\mathrm{b}$ & \\
\hline Schroederia setigera (SCHROED.) LEMMERM. & & $\mathrm{b}$ & $\mathrm{b}$ & \\
\hline Schroederia spiralis (PRINTZ) KORSHIKOV & & $\mathrm{ab}$ & $\mathrm{ab}$ & \\
\hline Selenastrum bibraianum REINSCH & $\mathrm{ab}$ & $\mathrm{ab}$ & $a b$ & $a b$ \\
\hline Selenastrum gracile REINSCH & $\mathrm{ab}$ & & & \\
\hline Siderocelopsis kolkwitzii (NAUMANN) HINDÁK & $\mathrm{ab}$ & & $a b$ & \\
\hline Siderocelis ornata (FоTт) FотT & $\mathrm{ab}$ & $\mathrm{ab}$ & $a b$ & $\mathrm{ab}$ \\
\hline Siderocelis pseudoblonga HINDÁK & & & a & \\
\hline Siderocelis sphaerica HINDÁK & & & a & \\
\hline Siderocystopsis fusca (KORSHIKOV) SwALE & $\mathrm{ab}$ & $\mathrm{ab}$ & $\mathrm{ab}$ & \\
\hline Siderocystopsis irregularis (HINDÁK) HINDÁK & & & $\mathrm{ab}$ & \\
\hline Tetrachlorella alternans (G. M. SмITH) KORSHIKOV & a & a & a & $\mathrm{a}$ \\
\hline Tetrachlorella ornata KORSHIKOV & & $\mathrm{b}$ & $\mathrm{b}$ & \\
\hline Tetraedron caudatum (CORDA) HANSG. & $\mathrm{ab}$ & $\mathrm{ab}$ & $a b$ & $\mathrm{ab}$ \\
\hline Tetraedron incus (TEILING) G. M. SMITH & $\mathrm{ab}$ & $\mathrm{ab}$ & $a b$ & \\
\hline Tetraedron mediocris HiNDÁK & & & $\mathrm{b}$ & \\
\hline Tetraedron minimum (A.K.H. BRAUN) HANSG. & a & & a & $\mathrm{a}$ \\
\hline Tetrastrum heteraxanthum (NORDST.) CHODAT & & & a & \\
\hline Tetrastrum komarekii HINDÁK & $\mathrm{ab}$ & & $a b$ & \\
\hline
\end{tabular}




\begin{tabular}{|c|c|c|c|c|}
\hline Cont. Table 3 & $\mathbf{O L}$ & $\mathbf{L A}$ & $\mathbf{D E}$ & DY \\
\hline Tetrastrum staurogeniaeforme (SCHRÖD.) LEMMERM. & $\mathrm{ab}$ & $\mathrm{ab}$ & $\mathrm{ab}$ & $\overline{a b}$ \\
\hline Tetrastrum triangulare (CHODAT) KOMÁREK & & & a & \\
\hline Treubaria triappendiculata $\mathrm{C}$. BERNARD & a & $\mathrm{a}$ & a & $\mathrm{a}$ \\
\hline Westella botryoides (W. WEST) DE WILD. & $\mathrm{ab}$ & $a b$ & $\mathrm{ab}$ & $a b$ \\
\hline \multicolumn{5}{|l|}{ Ulotrichales } \\
\hline Elakatothrix genevensis (REVERDIN) HINDÁK & a & & a & $\mathrm{a}$ \\
\hline Elakatothrix spirochroma (REVERDIN) HINDÁK & & & a & \\
\hline Elakatothrix subacuta KORSHIKOV & & a & a & $\mathrm{a}$ \\
\hline Geminella planctonica (BOLCH.) TIVARY ET PANDEY & & & $\mathrm{b}$ & \\
\hline Hortobagyiella verrucosa (HEYNIG) HINDÁK & & & $a b$ & \\
\hline Klebshormidium flaccidum (KüTZ.) P. C. SILVA et al. & & $\mathrm{ab}$ & & \\
\hline Koliella elongata (NYGAARD) NYGAARD & & & $\mathrm{b}$ & \\
\hline Koliella longiseta (VISCHER) HINDÁK & $\mathrm{ab}$ & & $\mathrm{ab}$ & \\
\hline Koliella spirotaenia (G. S. WEST) HINDÁK & & $a b$ & $\mathrm{ab}$ & \\
\hline Koliella variabilis (NYGAARD) HINDÁK & & & $\mathrm{a}$ & \\
\hline Marvania geminata HINDÁK & & $a b$ & $\mathrm{ab}$ & \\
\hline Planctonema lauterbornii SCHMIDLE & & & $\mathrm{ab}$ & \\
\hline Stichococcus contortus (CHODAT) HINDÁK & & $\mathrm{ab}$ & $a b$ & \\
\hline $\begin{array}{l}\text { Stichococcus pelagicus (NYGAARD) HINDÁK } \\
\text { CONJUGATOPHYCEAE }\end{array}$ & & $\mathrm{ab}$ & $\mathrm{ab}$ & $a b$ \\
\hline \multicolumn{5}{|l|}{ Zygnematales } \\
\hline Mougeotia sp. & & & $\mathrm{ab}$ & \\
\hline \multicolumn{5}{|l|}{ Desmidiales } \\
\hline Closterium acerosum (SCHROED.) EHRENB. & & & $\mathrm{ab}$ & \\
\hline Closterium acutum RALFS & & & $\mathrm{ab}$ & \\
\hline Closterium limneticum LEMMERM. & $\mathrm{ab}$ & $\mathrm{ab}$ & $\mathrm{ab}$ & $a b$ \\
\hline Closterium littorale GAY & & & $\mathrm{ab}$ & \\
\hline Closterium moniliferum (BORY) EHRENB. ex RALFS & $\mathrm{ab}$ & $\mathrm{ab}$ & $\mathrm{ab}$ & \\
\hline Closterium strigosum BRÉB. & & & $\mathrm{a}$ & \\
\hline Closterium spp. & & & $a b$ & \\
\hline Cosmarium formulosum LUND. & & & $\mathrm{b}$ & $\mathrm{b}$ \\
\hline Cosmarium moniliforme TURPIN EX RALFS & & $a b$ & $\mathrm{ab}$ & \\
\hline Cosmarium pygmaeum $\mathrm{W}$. ARCHER & & & a & \\
\hline Cosmarium spp. & $\mathrm{ab}$ & $\mathrm{ab}$ & $\mathrm{ab}$ & $a b$ \\
\hline Staurastrum chaetoceros (SCHRÖD.) G. M. SMITH & $\mathrm{ab}$ & $a b$ & $\mathrm{ab}$ & \\
\hline Staurastrum planctonicum TeILING & $\mathrm{ab}$ & $\mathrm{ab}$ & & \\
\hline Staurastrum spp. & $\mathrm{ab}$ & $a b$ & $\mathrm{ab}$ & $a b$ \\
\hline \multicolumn{5}{|l|}{ EUGLENOPHYCEAE } \\
\hline Colacium cyclopicola (GICKLH.) BOURR. & & & & $a b$ \\
\hline Colacium vesiculosum EHRENB. & $\mathrm{b}$ & & & \\
\hline Euglena acus EHRENB. var. acus & $\mathrm{ab}$ & & $\mathrm{ab}$ & $a b$ \\
\hline Euglena acus var. hyalina KLEBS & & & $\mathrm{b}$ & \\
\hline Euglena agilis H. J. CARTER & $\mathrm{ab}$ & $a b$ & $\mathrm{ab}$ & $a b$ \\
\hline Euglena caudata HÜBNER & $\mathrm{ab}$ & $\mathrm{ab}$ & $a b$ & $a b$ \\
\hline Euglena clara SKUJA & $\mathrm{b}$ & & & \\
\hline Euglena clavata SKUJA & $\mathrm{a}$ & & & \\
\hline Euglena ehrenbergii G.A. KLEBS & & & $\mathrm{ab}$ & $a b$ \\
\hline Euglena ignobilis JOHNSON & & & $\mathrm{b}$ & \\
\hline Euglena limnophila LEMMERM. & $\mathrm{ab}$ & $\mathrm{ab}$ & $\mathrm{ab}$ & \\
\hline Euglena mutabilis SCHMITZ & a & & & \\
\hline
\end{tabular}




\begin{tabular}{|c|c|c|c|c|}
\hline Cont. Table 3 & $\overline{\mathrm{OL}}$ & $\mathbf{L A}$ & $\mathbf{D E}$ & DY \\
\hline Euglena oxyuris SCHMARDA & & & $\mathrm{b}$ & \\
\hline Euglena rostrata SCHILLER & $\mathrm{b}$ & & $\mathrm{b}$ & \\
\hline Euglena rustica SCHILLER & & & & $\mathrm{b}$ \\
\hline Euglena spatirhyncha SKUJA & & & $\mathrm{b}$ & \\
\hline Euglena spirogyra EHRENB. & & $\mathrm{ab}$ & $a b$ & $a b$ \\
\hline Euglena splendens P.A. DANG. & & & a & \\
\hline Euglena texta (DUJARD.) HÜBNER & $a b$ & $\mathrm{ab}$ & $\mathrm{ab}$ & $a b$ \\
\hline Euglena tripteris (DUJARD.) G.A. KLEBS & & & $\mathrm{a}$ & \\
\hline Euglena velata $\mathrm{G}$. A. KLEBS & & & a & \\
\hline Euglena viridis EHRENB. & & & $\mathrm{ab}$ & $a b$ \\
\hline Lepocinclis glabra DREZEPOLSKI & & $\mathrm{b}$ & & \\
\hline Lepocinclis ovum (EHRENB.) MINKIEWICZ & & & a & \\
\hline Monomorphina pyrum (EHRENB.) MERESCHKOWSKY & & $\mathrm{ab}$ & $a b$ & $a b$ \\
\hline Phacus acuminatus STOKES & & & $\mathrm{b}$ & \\
\hline Phacus caudatus HÜBNER & $a b$ & $\mathrm{ab}$ & $a b$ & \\
\hline Phacus curvicauda SVIRENKO & $\mathrm{b}$ & & & $\mathrm{b}$ \\
\hline Phacus helicoides POCHMANN & & & $\mathrm{a}$ & $\mathrm{a}$ \\
\hline Phacus longicauda (EHRENB.) DUJARD. var. longicauda & a & a & a & $\mathrm{a}$ \\
\hline Phacus longicauda var. insecta HuB.-PEST. & & & & $\mathrm{b}$ \\
\hline Phacus longicauda var. tortus LEMMERM. & & & $\mathrm{b}$ & \\
\hline Phacus orbicularis HÜBNER & & a & & \\
\hline Phacus pleuronectes (EHRENB.) DUJARD. & $\mathrm{b}$ & $\mathrm{b}$ & $\mathrm{b}$ & $\mathrm{b}$ \\
\hline Phacus pusillus LEMMERM. & a & & & \\
\hline Phacus stokesii LEMMERM. & & & $\mathrm{b}$ & \\
\hline Phacus triqueter (EHRENB.) DURJADIN & & & $\mathrm{b}$ & \\
\hline Strombomonas eurystoma POPOVA & & & $a b$ & \\
\hline Strombomonas schauslandii (LEMMERM.) DEFLANDRE & & $\mathrm{ab}$ & $\mathrm{ab}$ & \\
\hline Trachelomonas cervicula STOKES & & & $\mathrm{b}$ & \\
\hline Trachelomonas hispida (PERTY) STEIN & a & a & & \\
\hline Trachelomonas irregularis SVIRENKO & $\mathrm{b}$ & & & \\
\hline Trachelomonas intermedia DANGEARD & & & a & \\
\hline Trachelomonas nigra SVIRENKO & $a b$ & $a b$ & $a b$ & $a b$ \\
\hline Trachelomonas oblonga LEMMERM. & & & $a b$ & $a b$ \\
\hline Trachelomonas ovalis (DADAY) LEMMERM. & & & $a b$ & $a b$ \\
\hline Trachelomonas planctonica SVIRENKO & $a b$ & $\mathrm{ab}$ & $a b$ & \\
\hline Trachelomonas scabratula (PLAYFAIR) DEFLANDRE & & & $\mathrm{b}$ & \\
\hline Trachelomonas volvocina EHRENB. & $a b$ & $a b$ & $\mathrm{ab}$ & $\mathrm{ab}$ \\
\hline
\end{tabular}

Table 4. Results of forward selection procedure of partial CCA, IF - inflation factor.

\begin{tabular}{llllllll}
\hline & \multicolumn{3}{c}{ Marginal effects } & \multicolumn{3}{c}{ Conditional effects } \\
& IF & Lambda 1 & F & P & Lambda A & F & P \\
\hline Conductivity & 1.37 & 0.19 & 1.78 & $0.008^{*}$ & 0.19 & 1.78 & $0.008^{*}$ \\
Temperature & 1.35 & 0.18 & 1.61 & $0.020^{*}$ & 0.15 & 1.43 & 0.182 \\
pH & 1.36 & 0.11 & 0.93 & 0.580 & 0.07 & 0.65 & 0.744 \\
\hline
\end{tabular}


Table 5. Number of taxa identified in the Morava and Dyje Rivers in May and August 2006.

\begin{tabular}{lcccccccccc}
\hline Groups & & & May & & & & \multicolumn{3}{c}{ August } \\
& OL & LA & DE & DY & total & OL & LA & DE & DY & total \\
\hline CYANOPHYCEAE & $\mathbf{2 4}$ & $\mathbf{3 3}$ & $\mathbf{3 6}$ & $\mathbf{2 0}$ & $\mathbf{4 5}$ & $\mathbf{3 3}$ & $\mathbf{3 6}$ & $\mathbf{4 2}$ & $\mathbf{2 6}$ & $\mathbf{5 3}$ \\
Chroococcales & 12 & 18 & 20 & 13 & 24 & 18 & 19 & 25 & 15 & 30 \\
Oscillatoriales & 9 & 8 & 8 & 4 & 10 & 10 & 9 & 8 & 6 & 12 \\
Nostocales & 3 & 7 & 8 & 3 & 9 & 5 & 8 & 9 & 5 & 11 \\
RHODOPHYCEAE & $\mathbf{0}$ & $\mathbf{0}$ & $\mathbf{0}$ & $\mathbf{0}$ & $\mathbf{0}$ & $\mathbf{0}$ & $\mathbf{1}$ & $\mathbf{0}$ & $\mathbf{0}$ & $\mathbf{1}$ \\
DINOPHYCEAE & $\mathbf{0}$ & $\mathbf{0}$ & $\mathbf{0}$ & $\mathbf{2}$ & $\mathbf{2}$ & $\mathbf{3}$ & $\mathbf{3}$ & $\mathbf{3}$ & $\mathbf{3}$ & $\mathbf{3}$ \\
CRYPTOPHYCEAE & $\mathbf{2}$ & $\mathbf{3}$ & $\mathbf{3}$ & $\mathbf{1}$ & $\mathbf{4}$ & $\mathbf{4}$ & $\mathbf{3}$ & $\mathbf{3}$ & $\mathbf{1}$ & $\mathbf{4}$ \\
CHRYSOPHYCEAE & $\mathbf{8}$ & $\mathbf{6}$ & $\mathbf{7}$ & $\mathbf{1}$ & $\mathbf{1 0}$ & $\mathbf{3}$ & $\mathbf{4}$ & $\mathbf{8}$ & $\mathbf{3}$ & $\mathbf{9}$ \\
BACILLARIOPHYCEAE & $\mathbf{1 1 8}$ & $\mathbf{1 1 5}$ & $\mathbf{8 6}$ & $\mathbf{8 7}$ & $\mathbf{1 7 1}$ & $\mathbf{1 3 4}$ & $\mathbf{8 0}$ & $\mathbf{1 3 3}$ & $\mathbf{6 6}$ & $\mathbf{1 8 9}$ \\
$\quad$ Coscinodiscales & 19 & 23 & 24 & 19 & 30 & 27 & 21 & 28 & 20 & 32 \\
$\quad$ Naviculales & 99 & 92 & 62 & 68 & 141 & 107 & 59 & 105 & 46 & 157 \\
XANTHOPHYCEAE & $\mathbf{2}$ & $\mathbf{0}$ & $\mathbf{1}$ & $\mathbf{0}$ & $\mathbf{3}$ & $\mathbf{2}$ & $\mathbf{2}$ & $\mathbf{7}$ & $\mathbf{2}$ & $\mathbf{8}$ \\
CHLOROPHYCEAE & $\mathbf{8 0}$ & $\mathbf{9 3}$ & $\mathbf{1 0 3}$ & $\mathbf{5 1}$ & $\mathbf{1 4 4}$ & $\mathbf{8 0}$ & $\mathbf{9 7}$ & $\mathbf{1 2 5}$ & $\mathbf{5 9}$ & $\mathbf{1 4 8}$ \\
$\quad$ Volvocales & 14 & 17 & 24 & 8 & 32 & 10 & 14 & 20 & 8 & 26 \\
Tetrasporales & 1 & 0 & 0 & 0 & 1 & 0 & 2 & 2 & 1 & 3 \\
Chlorococcales & 65 & 71 & 72 & 42 & 99 & 69 & 76 & 94 & 49 & 109 \\
$\quad$ Ulotrichales & 0 & 6 & 7 & 1 & 11 & 1 & 5 & 9 & 1 & 10 \\
CONJUGATOPHYCEAE & $\mathbf{5}$ & $\mathbf{3}$ & $\mathbf{2}$ & $\mathbf{4}$ & $\mathbf{9}$ & $\mathbf{6}$ & $\mathbf{7}$ & $\mathbf{1 2}$ & $\mathbf{4}$ & $\mathbf{1 3}$ \\
EUGLENOPHYCEAE & $\mathbf{1 7}$ & $\mathbf{1 6}$ & $\mathbf{1 5}$ & $\mathbf{3}$ & $\mathbf{2 9}$ & $\mathbf{1 5}$ & $\mathbf{1 3}$ & $\mathbf{2 9}$ & $\mathbf{1 7}$ & $\mathbf{4 1}$ \\
$\boldsymbol{\Sigma}$ & $\mathbf{2 5 6}$ & $\mathbf{2 6 9}$ & $\mathbf{2 5 3}$ & $\mathbf{1 6 9}$ & $\mathbf{4 1 7}$ & $\mathbf{2 8 0}$ & $\mathbf{2 4 6}$ & $\mathbf{3 6 2}$ & $\mathbf{1 8 1}$ & $\mathbf{4 6 8}$ \\
\hline
\end{tabular}

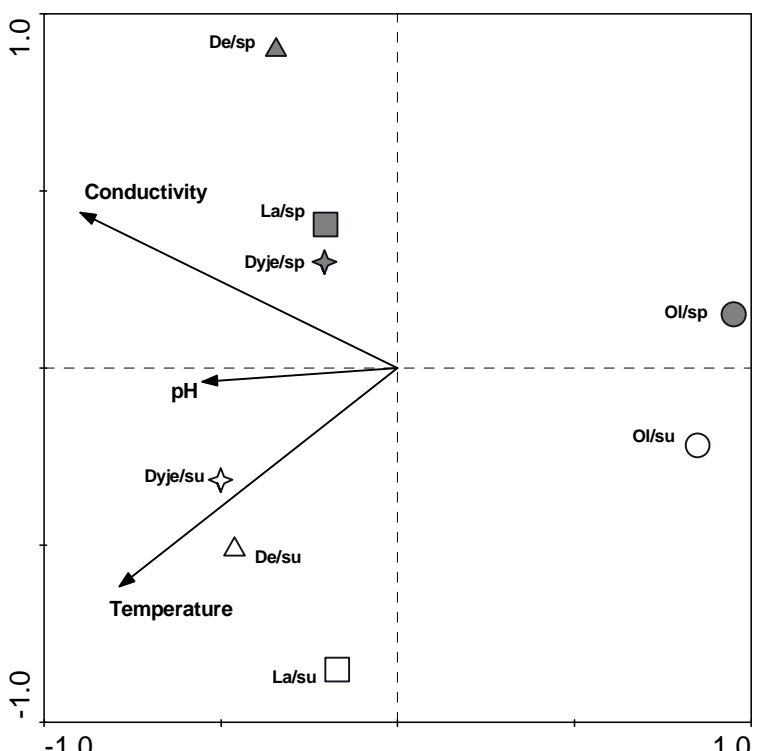

Fig. 4. Joint biplot diagram of samples vs. environmental variables (CCA): Ol - Morava at Olomouc, La - Morava at Lanžhot, De - Morava at Devín, Dyje - Dyje at Pohansko, sp - May sampling, $\mathrm{su}-$ August sampling.

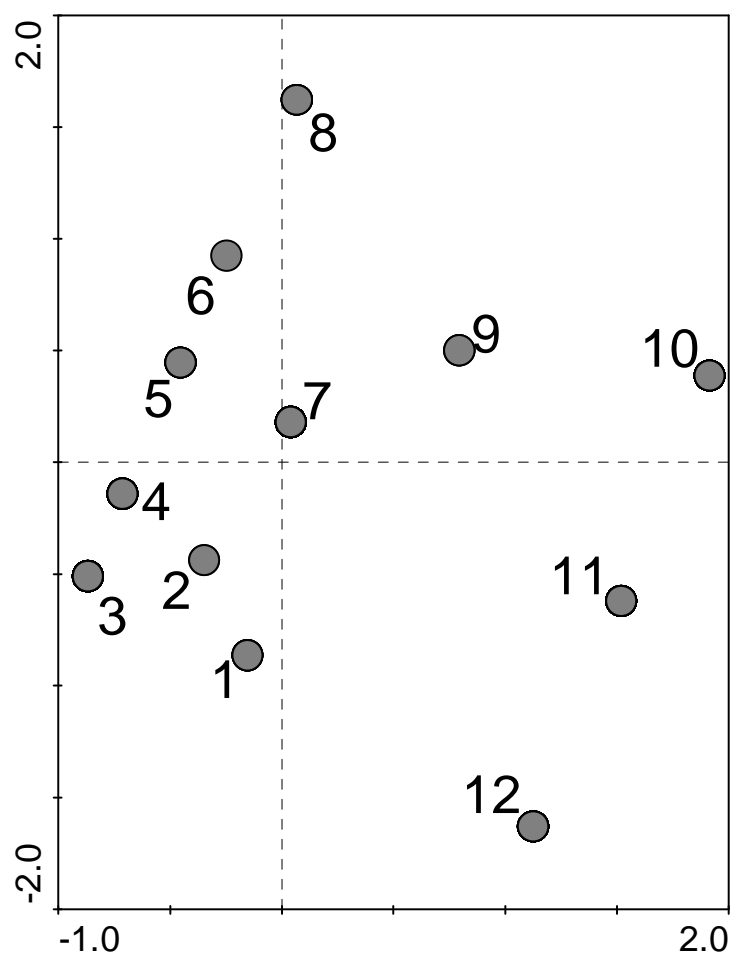

Fig. 5. Joint scatter plot of species (CCA): each number contains cluster of cyanobacteria and algae (Species Fit Range from 50 to 100\%); 
Species occurring at Olomouc more frequently than in the lower Morava stretch (species score 911): Achnanthes laevis, Gomphonema acuminatum, G. clavatum, Navicula atomus, Eunotia exigua, Fragilaria parasitica, Surirella ovalis, Pinnularia borealis, Chlamydomonas debaryana, C. incerta, Chlorogonium minimum, Euglena clara, E. clavata, Phacus pusillus, Trachelomonas irregularis;

Species commonly occurring at Lanžhot and Devín in spring (species score 5-6): Anabaena circinalis, Aphanocapsa parasitica, Chlamydomonas bicocca, $C$. bilatus, C. pertusa, C. skujae, Chloromonas anurae, Gloeomonas lateperforata, Gonium sociale, Haematococcus buetschlii, Pteromonas limnetica, Euglena splendens, E. tripteris, E. velata, Lepocinclis ovum, Phacus orbicularis and Trachelomonas intermedia;

Species commonly occurring at Lanžhot and Devín in summer (species score 1-4): Aphanocapsa grevillei, A. minima, Aphanothece bachmanii, Fragilaria nanana, Gomphonema insigne, Navicula erifuga, N. subplacentula, Surirella bifrons, Chlorangiopsis flos-aquae, Stylosphaeridium stipitatum, Coelastrum polychordum, Dicellula geminata, Didymogenes anomala, D. palatina, Granulocystis helenae, Tetrachlorella ornata, Koliella elongata, Euglena ignobilis, E. spatirhyncha, Lepocinclis glabra, Trachelomonas cervicula and $T$. scabratula;

Group of commonly occurring species (species score 7-8 and species under Fit Range 50\%): Chroococcus limneticus, Cyanogranis ferruginea, Microcystis aeruginosa, M. ichtyoblabe, Snowella litoralis, Woronichinia naegeliana, Planktothrix agardhii, Anabaena flos-aquae, Acanthosphaera zachariasii, Actinastrum hantzschii, Chlorella vulgaris, Coelastrum astroideum, C. microporum, Coenochloris planctonica, Coenococcus planctonicus, Crucigeniella apiculata, Dictyosphaerium tetrachotomum, Franceia echidna, F. tenuispina, Monoraphidium contortum, Oocystella lacustris, Pediastrum boryanum, P. duplex, P. simplex, Planktosphaeria gelatinosa, Scenedesmus spp., Siderocelis ornata.

\section{Discussion}

A wide spectrum of cyanobacteria and algae was identified during our study on the main parts of the Morava River and the most important rightside tributary the Dyje River. As follows from previous studies (HINDÁK \& HINDÁKOVÁ 1997, 1998, 2004, MARVAN et al. 2004) phytoplankton of the Morava River has been changing. In 1950 's, when organic pollution developed, high biomass and waterbloom of Microcystis spp. occurred, while at present time the amount of cyanobacteria is not so high. Thus, we did not record dense waterbloom, even if 53 species of cyanobacteria were found through investigated sites. According to HINDÁK's study (2004) in the lowermost Morava River stretch at Devín in 2003 was found 42 species of cyanobacteria, which is similar to our investigations. However, a significant influence on biomass and diversity of cyanobacteria have had Nové Mlýny dams, which usually produce a strong population of Microcystis species and recently recorded in the end of 1990's. According to HINDÁKOVÁ (1994) the most abundant species of centric diatoms were found in the lowermost Morava River stretch Aulacoseira granulata, Cyclotella meneghiniana and Stephanodiscus binderanus. We found at sampling site at Devín 28 species of centric diatoms, while in 1994 had been determined only 18 species. In case of pennate diatoms, we recorded 110 species in contrast to Hindáková who had determined 117 species. With respect to summer flood in the beginning of August 2006 the diversity of pennate diatoms increased in Olomouc and Devín as benthic species were drifted. Number of chlorococcal algae, as found in 2006, was lower than in 2003. High instantaneous discharge in the investigated rivers caused dilution of phytoplankton and consequently lacking of rare species. As follows from previous HINDÁKs' studies (1977, 1980, 1984, 1988, 1990) such rare species of green algae as Danubia ansa and Crucigeniopsis divergens were not confirmed. On the other hand, Coenochloris astroidea was recorded at Devín, but at Olomouc was found for the first time.

With respect to last investigation on the phytoplankton of the Morava River in autumn 2005 , which was identically sampled, the species diversity is different to our study and moreover strongly influenced by sampling period (HINDÁK et. al 2006). The diversity of 346 species and infraspecific taxa had been found in the Morava and Dyje Rivers, while at the same localities in summer 2006 were recorded 468 species. A discernible pattern of changes in the phytoplankton structure is possible to see in diatoms, which frequently occurred in spring 2006 and autumn 2005, while cyanobacteria and green algae dominate summer 2006. Diversity among other groups both Euglenophyceae and Conjugatophyce incresed with higher temperature in summer, while low temperature in 
autumn 2005 caused loss of diversity. The rest of algal flora containing groups such as Chrysophyceae, Cryptophyceae, Dinophyceae, Xanthophyceae and Ulotrichales did not show any marked changes of spieces richness amon sampling sites during our study as well as in the autumn phytoplankton. As follows from previous paper (Hindák et al. 2006) and with respect to findings the Morava River forms two major parts concerning the lowermost and middle stretch (from Lanžhot to Devín) and the upper stretch (at Olomouc), which is more similar to the Dyje River near its confluence with the Morava at Pohansko. Such measured environmental variables as temperature, conductivity and average discharge have markedly influenced structure and abundance of phytoplankton than $\mathrm{pH}$ or dissolved oxygen. The species richness in the Dyje River as a typical regulated lowland river is strongly influenced by Nové Mlýny dams and so we found lower diversity similar to this at Olomouc than at Lanžhot or Devín. Thus, there is no doubt that the large reservoirs significantly change phytoplankton structure of rivers with respect to former state. It means that we should not expect similar data to less or no influenced rivers in the same area.

Generally, with respect to our analysis of phytoplankton the Morava River seem to be rich in diversity of algae and especially in the lower river stretch. Wide research on phytoplankton has been actually made in the main Slovak rivers concerning the Danube, Váh, Hron, Ipel' and Slovak part of the Morava, which were found to be an important source of algal biodiversity in the territory of Slovakia (HINDÁK et al. 2002). Similar to our investigation, in the main Slovak rivers such species of cyanobacteria and algae as Microcystis aeruginosa, Planktothrix agardhii, Anabaena flos-aquae, Chrysococcus spp., Mallomonas spp., Cyclotella meneghiniana, Cyclostephanos dubius, Stephanodiscus hantzschii, Thalassiosira pseudonana, $T$. weissflogii were recorded as well as Carteria globosa, C. radiosa, Chlamydomonas incerta, Chl. monadina, Actinastrum hantzschii, Micractinium spp., Pediastrum spp. or Scenedesmus spp. etc. In Czech Republic the Lužnice River was investigated by PITHART et al. (1996) who found lower diversity in the phytoplankton but with frequent occurrence of species as mentioned from Slovak rivers. The broadest research on phytoplankton in the whole Europe was undoubtedly made in the Danube River from Germany to Ukraine and five to ten times higher biodiversity through the all taxonomic groups was observed (KUSELFETZMANN 1998).

Summarizing all known data, the Morava River belongs to the most important river systems in the Czech and Slovak Republics concerning unique spectrum of cyanobacteria and algae.

\section{Acknowledgements}

This study is a part of the VEGA projects Nos 2/4033/04 and 2/7069/27 supported by the Slovak Academy of Sciences, APVT-51-009102 supported by the Ministry of Education SR (F.H., A.H.) and by the Ministry of Agriculture CR grant NPGZ-M/03023. The authors are obliged to Miss Annie Brasseur in Sacramento, California, USA, and Mrs Jeannine Braem in Schoten, Belgium, for correcting the English. The authors are obliged to Ing. Milena Forejtníková (Water Research Institute, Brno, grant MŽP -VaV/650/3/03) for chemical data.

\section{References}

HAŠLER, P. \& PoulíčKovÁ, A. (2002): Planktic cyanobacteria of the Central and Northern Moravia. Czech Phycology 2: 25-32.

Hein, T., Baranyi, C., Heiler, G., Holarek, C., Riedler, P. \& SCHIEMER, F. (1999): Hydrology as a major factor determining plankton development in two floodplain segments and the River Danube, Austria.- Arch. Hydrobiol. 3: 439-452.

HeteŠA, J. \& MARvan, P. (eds)(1984): Biology of a newly impounded reservoir [Biologie nově napuštěné nádrže]. - Studie ČSAV, Praha, 3: 1-176 pp.

Heteša, J. \& MARVAN, P. (1987): Phytoplankton of streams of the lower part of the Morava River Basin [Fitoplankton vodotokov nižnej časti bassejna $r$. Moravy]. - In: Hejný, S. \& Romanenko, V. D. (eds): Hydrobiological studies of the Danube and its water reservoirs [Gidrobiologičeskie issledovanija Dunaja i pridunajskich vodojemov]. Sborn. nauč. trudov, Kiev, Naukova Dumka, 57-73 pp.

HetešA, J. \& MARVAN, P. (1997): Phytoplankton primary production in the Dyje River below Nové Mlýny reservoirs. - Acta Mus.Moraviae, Brno, Sci. biol. 82: $19-28$.

HindÁK, F. (1977): Studies on the chlorococcal algae (Chlorophyceae). I. - Biol. práce, Veda, Bratislava, 23/4: $1-192$.

HindÁK, F. (1980): Studies on the chlorococcal algae (Chlorophyceae). II. - Biol. práce, Veda, Bratislava, 26/6: 1-196.

HindÁK, F. (1984): Studies on the chlorococcal algae (Chlorophyceae). III. - Biol. práce, Veda, Bratislava, 30/1: 1-310.

HindÁK, F. (1988): Studies on the chlorococcal algae (Chlorophyceae). IV. - Biol. práce, Veda, Bratislava, 34/1-2: 1-264. 
HiNDÁK, F. (1990): Studies on the chlorococcal algae (Chlorophyceae). V. - Biol. práce, Veda, Bratislava, 23/4: 1-192.

HiNDÁK, F. (1996): Klúč na určovanie nerozkonárených vláknitých zelených rias (Ulotrichineae, Ulotrichales, Chlorophyceae) [Key to the unbranched filamentous green algae (Ulotrichineae, Ulotrichales, Chlorophyceae)]. - Bulletin SBS pri SAV, Bratislava, Supl. 1: 1-77.

HiNDÁK, F. (2001): Fotografický atlas mikroskopických siníc. [Photographic Atlas of Microscopic Cyanophytes] - 128 pp., Veda, Bratislava.

HindÁk, F. \& HindákovÁ, A. (1997): Cyanophytes and algae of the inundation lakes Stará Morava at Devín [Sinice a riasy inundačných jazier Stará Morava v Devíne]. - In: FerÁKOVÁ, V. (ed.): Flora, geology and paleontology of the National Natural Reserve Devínska Kobyla [Flóra, geológia a paleontológia Národnej prírodnej rezervácie Devínska Kobyla], APOP, Bratislava, 36-57 pp.

HINDÁK, F. \& HiNdÁKovÁ, A. (1998): Cyanophytes/Cyanobacteria and Algae [Sinice/cyanobaktérie a riasy]. - In: MARHOLD, K. \& HINDÁK, F. (eds): Checklist of nonvascular and vascular plants of Slovakia [Zoznam nižších a vyšších rastlín Slovenska], VEDA, Bratislava, 11-100 pp.

HindÁK, F. \& HindÁKOVÁ, A. (2004): Diversity of the phytoplankton of the Morava and Danube Rivers in Bratislava (W Slovakia) in 2003 [Diverzita fytoplanktónu rieky Moravy a Dunaja v Bratislave v r. 2003]. - Bull. Slov. Bot. Spočn., Bratislava, 26: 9-17.

HindÁk, F., HindákovÁ, A., Makovinská, J., Tothová, L. \& ElexovÁ, E. (2002): Biodiverzita a biomasa fytoplanktónu rieky Ipel’v r.1992-2001.-Bull. Slov. Bot. Spoločn. 24: 15-23.

Hindák, F., Hindáková, A., Marvan, P., Heteša, J. \& HAŠLER, P. (2006): Diversity, abundance and volume biomass of the phytoplankton of the Morava River (Czech Republic, Slovakia) and the Dyje River (Czech Republic) in November 2005.- Czech Phycology 6: 77-97.

HiNDÁK, F. \& MAKOvinSKÁ, J. (1996): Use of algae for monitoring rivers in Slovakia. - In: WhiTTON, B. A. \& RoTT, E. (eds): Use of algae for monitoring rivers II, Innsbruck, 133-136 pp.

HindÁKOVÁ, A. (1994): Planktic diatoms of the river Morava at Bratislava-Devín, Slovakia. - Ekológia, Bratislava, 13/Suppl. 1: 37-42.

Huber-Pestalozzi G. (1955): Euglenophyceen. - Die Binnengewässer, Stuttgart, 16/4: 1-606 pp.

KomáreK, J. \& ANAGnostidis, K. (1998): Cyanoprokaryota 1. Teil Chroococcales. - Süßwasserflora von Mitteleuropa, Jena - Stuttgart - Lübeck - Ulm, 19/1: 1-548 pp.
Kopf, W., Pöhlmann, W. \& Reimann, K. (1988): Grundlagen der Eutrophierung von Fliessgewässern, dargestellt am Beispiel von Main und Regnitz, 2 parts.- 623 pp., Bayerische Landesanstalt Wasserforschung, München.

Krammer, K. \& Lange-Bertalot, H. (1986): Bacillariophyceae, 1. Teil: Naviculaceae. - Süßwasserflora von Mitteleuropa, Stuttgart - Jena, 2/1: $1-876 \mathrm{pp}$.

Krammer, K. \& Lange-Bertalot, H. (1988): Bacillariophyceae, 2. Teil: Bacillariaceae, Epithemiaceae, Surirellaceae. - Süßwasserflora von Mitteleuropa, Stuttgart - Jena, 2/2: 1-596 pp.

Krammer, K. \& LANGE-Bertalot, H. (1991a): Bacillariophyceae, 3. Teil: Centrales, Fragilariaceae, Eunotiaceae. - Süßwasserflora von Mitteleuropa, Stuttgart - Jena, 2/3: 1-576 pp.

KRAMMER, K. \& LANGE-BeRTAlOT, H. (1991b): Bacillariophyceae, 4. Teil: Achnanthaceae, Kritische Ergänzungen zu Navicula (Lineolatae) und Gomphonema. - Süßwasserflora von Mitteleuropa, Stuttgart - Jena, 12/4: 1-437 pp.

Kusel-FetzMAnN, E. (1998): Das Phytoplankton. - In: Kusel-Fetzmann, E., Naidenow, W. \& Russev, B. (eds): Plankton und Benthos der Donau, Ergebnisse der Donau-Forschung, IAD Wien, 4: 1-376 pp.

MARvan, P. \& HeteŠA, J. (2000): Micro- and macrovegetation of cut-off arms of River Morava; [Mikro- a makrovegetace odstavených ramen řeky Moravy]. - In: PIthaRT, D. (ed.): Ecology of floodplain pools and river arms [Ekologie aluviálních tůní a říčních ramen]. Proc. Conf., Lužnice at Třeboň, 53-57 pp.

Marvan, P., Heteša, J., Hindák, F. \& Hindáková, A. (2004): Phytoplankton of the Morava river (Czech Republic, Slovakia): past and present. - Oceanological and Hydrobiological Studies, Gdańsk, 33/4: 42-60.

Pithart, D., Elster, J., Komárek, O. \& KlabouchovÁ, A. (1996): Microphyte vegetation.- In: PRACH, K., JENíK, J. \& LARGE, A.R.G. (eds.): Floodplain ecology and management, the Lužnice River in the Třebon̆ Biosphere Reserve, Central Europe, Academic Publishing, Amsterdam, 99-112 pp.

TER BraAK, C. J. F. \& ŠMilauer, P. (1998): Canoco references manual and user's guide to canoco for Windows. - 353 pp., Centre of Biometry, Wageningen,

(C) Czech Phycological Society (2007)

Received December 5, 2006

Aaccepted February 27, 2007 